This is the post peer-review accepted manuscript of:

F. Guidi, A. Guerra and D. Dardari, "Personal Mobile Radars with Millimeter-Wave Massive Arrays for Indoor Mapping," in IEEE Transactions on Mobile Computing, vol. 15, no. 6, pp. 1471-1484, 1 June 2016. https://doi.org/10.1109/TMC.2015.2467373

The published version is available online at: https://ieeexplore.ieee.org/document/7192657

C 2015 IEEE. Personal use of this material is permitted. Permission from IEEE must be obtained for all other uses, in any current or future media, including reprinting/republishing this material for advertising or promotional purposes, creating new collective works, for resale or redistribution to servers or lists, or reuse of any copyrighted component of this work in other works 


\title{
Personal Mobile Radars with Millimeter-Wave Massive Arrays for Indoor Mapping
}

\author{
Francesco Guidi, Member, IEEE, Anna Guerra, Student, IEEE, \\ Davide Dardari Senior Member, IEEE
}

\begin{abstract}
The adoption of millimeter-wave technology could open the possibility to integrate massive antenna arrays inside future $5 \mathrm{G}$ user mobile devices, with the possibility to enable new interesting applications. Within this context, in this paper we put forth the concept of a personal mobile radar operating at millimeter-waves and consisting of a massive array for accurate environmental mapping. Frequency selectivity and phase quantization effects are accounted for to characterize the achievable angle and range resolution necessary to collect environmental information. Successively, we propose an effective grid-based Bayesian mapping approach by introducing a new state-space model, which profits of the beneficial effects of the massive antenna array characteristics. Numerical results show that the idea herein investigated is feasible, and that a significant mapping performance is attainable even employing coarse antenna arrays provided that the number of antenna elements is sufficiently high.
\end{abstract}

Index Terms-Personal radar, indoor mapping, millimeter-wave, massive antenna arrays

\section{INTRODUCTION}

W ITHIN the concept of the Internet of Things, objects and devices are expected to be connected and mapped into the Internet space to enable environmentrelated applications [1]. In this context one key step is the digital representation (mapping) of the physical world into the so called smart space (i.e. a space that enables the cooperation and interaction among smart objects). While in the last years this process has rapidly taken place in outdoor environments thanks to the availability of GPS information and the introduction of locationbased applications, other techniques have to be adopted in indoor environments [2], [3], whereas manual mapping and updating of all buildings in the world would

- Francesco Guidi is with CEA, LETI, MINATEC Campus, 38054 Grenoble, France and at the time of submission he was with DEI, University of Bologna, Italy ,Via Venezia 52, 47521 Cesena, ITALY.

Anna Guerra and Davide Dardari are with the Dipartimento di Ingegneria dell'Energia Elettrica e dell'Informazione "Guglielmo Marconi" - DEI, University of Bologna,Via Venezia 52, 47521 Cesena, ITALY.

(e-mail: francesco.guidi@cea.fr, \{anna.guerra3,davide.dardari\}@unibo.it).

This research was supported by the Italian Ministerial PRIN project GRETA (Grant 2010WHY5PR) and in part by the H2020 IF-EF Marie-Curie project MAPS (Grant 659067).

This paper was presented, in part, at the IEEE Int. Conf. on Commun. (ICC), Sydney, Jun. 2014, and at the IEEE Asilomar Conf. on Signals, Systems, and Computers, Pacific Grove, USA, Nov. 2014.

Digital Object Identifier no. 10.1109/TMC.2015.2467373

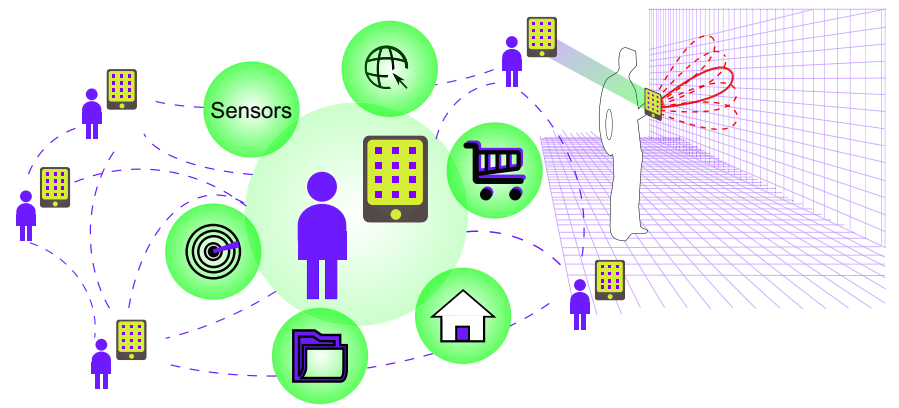

Fig. 1: Users, equipped with massive arrays on their smartphones, in participatory or not manner scan indoor environments with the opportunity to share their maps.

require an unaffordable cost and effort. Traditionally, simultaneous localization and mapping (SLAM) is based on the concept that a robot, moving in an unknown environment, recognizes the surrounding objects being then able to reconstruct a 2D/3D map of the area. Current accurate SLAM, for automatic creation of indoor maps [4]-[6], requires both high-definition distance estimates (ranging) and very narrow steering beams (angle resolution), traditionally accomplished by laser technology. In addition, the exploitation of WiFi and sensors integrated in smartphones for zero-effort automatic indoor positioning and mapping applications relate very poorly to the actual mobile position, and topological environment information can be hardly inferred.

Looking one step ahead, of particular interest for next generation (5G) mobile wireless communications is the millimeter-wave ( $\mathrm{mmW}$ ) technology, for example that working in the $60 \mathrm{GHz}$ band, which allows the exploitation of a large clean unlicensed bandwidth (up to $7 \mathrm{GHz}$ ) [7]-[10]. The reduced wavelength at $\mathrm{mmW}$ paves the way for packing massive arrays with electronic steering capabilities into a small area, as done in [11]. Authors in [12] studied how to realize $\mathrm{mmW}$ massive arrays, with dimensions which are becoming closer and closer similar to that of a tablet, even though several technological issues have still to be solved, such as energy consumption.

The idea to adopt $\mathrm{mmW}$ radars to overcome the short- 
comings of laser is not new and it has been considered for outdoor applications in some previous works, such as [13]-[16], where a dedicated very high-directional antenna is mechanically steered. The consequent nearpencil beam returns a precise angle and range information thus making the modeling and characterization of the environment with $\mathrm{mmW}$ radars very similar to that based on laser. Obviously, such systems can not be integrated into smartphones or tablets.

Stimulated by this possibility, in this paper we put forth the idea of placing massive antenna arrays in smartphones or tablets thus realizing a high-definition and low-cost personal mobile radar [17]-[19]. In fact, the availability of a large number of antennas on mobile devices opens the possibility of realizing narrow beams with electronic-driven steering capabilities that are fundamental for high-accuracy mapping as a cheap, energy efficient alternative to laser technology (see Fig. 1) [20]. In our vision the pervasive availability of personal radar capabilities on smartphones will enable high-definition, zero-effort and automatic indoor building mapping by exploiting the possibility of crowd sourcing (crowd mapping) [21]-[24]. In addition, other new applications are possible such as blind people aid or the pictorial 3D environmental mapping where the third dimension results by the integration of the angle/range information collected by the personal radar with the 2D picture coming from the conventional embedded camera. From a complementary perspective, in [25], a system of stereo cameras and integrated sensors is used to have the depth information and to create a 3D image of the environment. The main drawback of this solution is that cameras are not able neither to automatically scan the environment as they do not perform a beamforming operation (i.e. the user must be participatory as he/she has to "pilot" the scan process by properly moving the camera) nor to retrieve information about the electromagnetic properties of materials. Our approach outperforms these weakness thanks to the adoption of $\mathrm{mmW}$ technology and of a multi-antenna radar system, which can scan the environment even if the smartphone is kept in the user pocket, i.e. without having the user participatory.

In our proposed $\mathrm{mmW}$ personal radar, wideband signals are employed to assure high ranging performance [26]. Unfortunately, technology constraints prevent the use of wideband time-delay components necessary to realize the required signals alignment from different array elements and only simple digitally-controlled phase shifters can be realistically adopted. Therefore, loss in steering and ranging accuracy due to quantized phase shifters and frequency selectivity might arise [27], [28]. In addition, even in case massive arrays are adopted, laserlike beams are not obtainable and each measurement might be affected by contributions coming from different directions due to the effect of an increased main lobe width and side-lobes level in the antenna radiation pattern. Thus, dedicated mapping approaches are needed to overcome these limitations and to make massive arrays an attractive candidate for environment mapping. To validate our idea, the main contributions of the paper can be summarized as follows:

- We analyse the steering and ranging capabilities of the proposed $\mathrm{mmW}$ personal radar concept as a function of the signal bandwidth and the number of antennas composing the array. Specifically, the impact of frequency selectivity and quantization phase errors on the beam pointing error and beam width spread is jointly characterized.

- We propose a low-complexity personal radar transceiver employing a massive antenna array and based on the transmission of wideband signals with non-coherent processing of the corresponding backscatter response of the environment. Our approach is different from the current state-of-theart. In fact, a classic two-step approach is typically followed [15], [16]: first, for each test direction the obstacle is detected and its distance measured and, subsequently, this "hard" decision becomes the input for the mapping algorithm. Differently, we propose a new observation model that considers all the available raw measurements ("soft" decision) and hence fully exploits all the information coming from the antenna array.

- The environment mapping capabilities of the personal radar are investigated by introducing a new Bayesian state-space model which considers a grid representation of the environment based on the electromagnetic characteristics of each space element and incorporating the new observation model able to properly account for the intrinsic characteristic of the massive antenna array and scanning process.

The rest of the paper is organized as follows. The impact of errors on pointing and angle resolution in massive antenna arrays due to frequency selectivity and phase quantization noise is characterized in Sec. 2 and 3, where the trade-off between ranging and steering accuracy as a function of the number of elements composing the antenna array is also investigated. The personal radar mapping capabilities are modeled and characterized in Sec. 4 , whereas in Sec. 5 numerical results related to a typical indoor scenario are provided. Finally, the conclusions are drawn in Sec. 6.

\section{Sources of Error in Massive Antenna ARRAYS}

\subsection{Planar Phased Arrays}

The planar array configuration appears well suited for massive antennas array integration in smartphones and tablets. Consider the scheme reported in Fig. 2, where $N_{\text {array }}=M N$ antenna elements are placed in a rectangular area of dimension $L_{\mathrm{x}} \times L_{\mathrm{y}}$, with $L_{\mathrm{x}}=M d_{\mathrm{x}}$ and $L_{\mathrm{y}}=N d_{\mathrm{y}}$. The elements are spaced apart of $d_{\mathrm{x}}\left(d_{\mathrm{y}}\right)$ in the $x(y)$ dimension. According to [29], and considering a uniform array, the array factor (AF) at frequency $f$ is 
defined as ${ }^{1}$

$$
\operatorname{AF}(\Theta, f)=\sum_{m=1}^{M} \sum_{n=1}^{N} e^{j\left[(m-1) \Psi_{x}+(n-1) \Psi_{y}\right]}
$$

with

$$
\begin{aligned}
& \Psi_{\mathrm{x}}=k d_{\mathrm{x}} \sin (\theta) \cos (\phi)+\beta_{\mathrm{x}} \\
& \Psi_{\mathrm{y}}=k d_{\mathrm{y}} \sin (\theta) \sin (\phi)+\beta_{\mathrm{y}}
\end{aligned}
$$

with $\Theta=(\theta, \phi)$ being the considered direction identified by the elevation and the azimuthal angles, $\theta$ and $\phi$, respectively, and $k=2 \pi f / c$ the wavenumber, with $c$ indicating the speed of light. To steer the main lobe towards a specific direction $\Theta_{0}$, parameters $\beta_{\mathrm{x}}$ and $\beta_{\mathrm{y}}$ have to be set to

$$
\begin{aligned}
& \beta_{x}=-k_{0} d_{x} \sin \left(\theta_{0}\right) \cos \left(\phi_{0}\right) \\
& \beta_{y}=-k_{0} d_{y} \sin \left(\theta_{0}\right) \sin \left(\phi_{0}\right)
\end{aligned}
$$

with $k_{0}=2 \pi f_{0} / c$, and $f_{0}$ being the reference frequency for which the steering parameters are designed. In the steering direction, for $f=f_{0}$ and in the absence of array non-idealities, it is $\operatorname{AF}\left(\Theta_{0}, f_{0}\right)=N_{\text {array }}$.

The complex beam-steering weights determining the beam shape and pointing direction can be expressed as

$$
\omega_{m n}=e^{j \varphi_{m n}}=e^{j\left[(m-1) \beta_{x}+(n-1) \beta_{y}\right]}
$$

where $\varphi_{m n}$ is the weight phase, and $\beta_{\mathrm{x}}$ and $\beta_{\mathrm{y}}$ are defined in (3).

\subsection{Sources of Error in Antenna Arrays}

There are several issues that arise when massive wideband phased arrays are adopted and both accurate beam steering and ranging have to be guaranteed. They are even more pronounced when working at very high frequencies due to technological and cost constraints [30].

This section deals with the effects of phase quantization errors and large signal bandwidth. The joint impact of such sources of error results in beam pointing errors and beamwidth spread that must be properly characterized when analyzing the performance of mapping schemes, as done in Sec. 4.

\subsubsection{Large Signal Bandwidth}

A large bandwidth is in general desirable thanks to the corresponding achievable high ranging resolution [31]. However, in a wideband system, the received signal arrives at each antenna element with a delay that is not negligible compared to the signal duration, and hence it cannot be compensated by adopting only phase shifters, as typically done in narrowband systems. Unfortunately, the adoption of a huge number of time delay circuits represents a high-cost solution, especially at millimeter frequencies, then phase shifters remain at the moment

1. For simplicity and without loss of generality, we consider isotropic array elements.
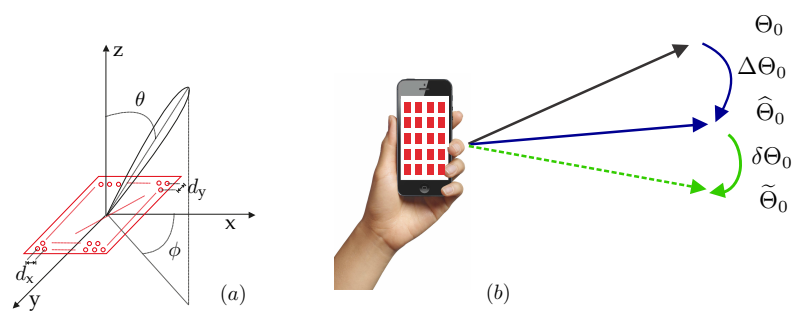

Fig. 2: Array structure (a) and beam pointing error scheme (b).

the most viable solution [32]. Consequently, in the absence of time delays, the accumulation of the component received by each branch will result in a signal shape distortion. In other words, the actual steering direction and beamwidth would depend on frequency (beam squinting effect). For this reason the effects of signal bandwidth have to be carefully accounted for.

\subsubsection{Array Weights Errors}

Even the adoption of high resolution analog phase shifters might still not be a feasible solution for $\mathrm{mmW}$ massive antenna arrays if we aim at keeping a low system cost. Digitally controlled phase shifters implementing a discrete set of phase shifts represent a cheaper alternative at the expense of quantization errors [20]. Some new solutions have been investigated in [30] to find a compromise between the number of elements and the phase shifters accuracy.

Starting from the general model for the quantized version $\tilde{\omega}_{m n}$ of weights $\omega_{m n}$ proposed in [28], we express $\tilde{\omega}_{m n}=\omega_{m n} e^{j \delta_{m n}}$, with $\delta_{m n} \sim \mathcal{U}(-\Delta / 2, \Delta / 2)$ uniformly distributed random variables (RVs) in the quantization step $\Delta$. Note that array weights errors affect the beam steering accuracy (see Fig. 2) and, consequently, the beamwidth characteristics. In addition, the misalignment of pulses at each array element causes temporal spread if it is not well counteracted. These effects can reduce the angle and ranging resolution as it will be detailed in the next section.

\section{IMPACt OF ERRORS ON POINTING AND AN- GLE RESOLUTION}

We now investigate the impact of errors on the array characteristics. In particular, denote with $\widetilde{\mathrm{AF}}(\Theta, f)$ the actual AF in the presence of the aforementioned errors. Expression (1) becomes

$$
\widetilde{\mathrm{AF}}(\Theta, f)=\sum_{m=1}^{M} \sum_{n=1}^{N} e^{j\left[(m-1) \Psi_{x}+(n-1) \Psi_{y}\right]} e^{j \delta_{m n}}
$$

where $\delta_{m n}$ 's are the phase errors previously defined.

Now denote with $\widetilde{\Theta}_{0}=\left(\tilde{\theta}_{0}, \tilde{\phi}_{0}\right)$ the actual beam pointing direction at frequency $f$ and in presence of arrays non idealities. We can relate the actual beam pointing error to the desired beam pointing direction as $\widetilde{\Theta}_{0}=\Theta_{0}+\Delta \widehat{\Theta}_{0}+\delta \Theta_{0}$, with $\Delta \widehat{\Theta}_{0}+\delta \Theta_{0}$ indicating the shift from the desired pointing direction consisting of both a 
deterministic $\Delta \widehat{\Theta}_{0}$ and random quantity $\delta \Theta_{0}=\left(\delta \theta_{0}, \delta \phi_{0}\right)$ due to frequency selectivity and phase quantization, respectively (Fig. 2). Note that $\widehat{\Theta}_{0}=\left(\hat{\theta}_{0}, \hat{\phi}_{0}\right)=\Theta_{0}+\Delta \widehat{\Theta}_{0}$ is the actual beam pointing direction at frequency $f$ and in absence of quantization error, with

$$
\hat{\theta}_{0}=\sin ^{-1}\left(\frac{f_{0}}{f} \sin \left(\theta_{0}\right)\right), \quad \hat{\phi}_{0}=\phi_{0} .
$$

For what follows it is convenient to derive the squared $\mathrm{AF}$

$$
\begin{aligned}
|\widetilde{\mathrm{AF}}(\Theta, f)|^{2} & =\sum_{m n p q} e^{j\left[(m-p) \Psi_{x}+(n-q) \Psi_{y}\right]} e^{j\left(\delta_{m n}-\delta_{p q}\right)} \\
& =|\operatorname{AF}(\Theta, f)|^{2}+\zeta_{\mathrm{err}}(\Theta, f)
\end{aligned}
$$

with

$$
\zeta_{\mathrm{err}}(\Theta, f)=\sum_{m n p q} e^{j\left[(m-p) \Psi_{x}+(n-q) \Psi_{y}\right]}\left[e^{j\left(\delta_{m n}-\delta_{p q}\right)}-1\right]
$$

and $\sum_{m n p q}=\sum_{m}^{M} \sum_{n}^{N} \sum_{p}^{M} \sum_{q}^{N}$. Equation (7) puts in evidence the deterministic and random terms composing the squared AF.

Considering $\delta_{m n}$ 's as independent, identically distributed (i.i.d.) RVs, the average squared $\mathrm{AF}$ is

$$
\mathbb{E}\left\{|\widetilde{\mathrm{AF}}(\Theta, f)|^{2}\right\}=N_{\text {array }}+\left(|\mathrm{AF}(\Theta, f)|^{2}-N_{\text {array }}\right) \chi^{2}
$$

where $\chi=\mathbb{E}\left\{e^{j \delta_{m n}}\right\}=\frac{2}{\Delta} \sin \left(\frac{\Delta}{2}\right)$.

In the following we will discuss about the two effects that arise when phase errors and frequency selectivity are present. First, the beam pointing error phenomenon is investigated. Second, we address the beamwidth deviations caused by the joint influence of phase errors and squint effect.

\subsection{Beam Pointing Error}

We now evaluate the impact of phase errors on the beam pointing direction. In particular we derive the mean and the variance of the mean beam pointing error.

The mean square error (MSE) of the beam pointing error is given by

$\operatorname{MSE}_{\theta}\left(f, \theta_{0}\right)=\mathbb{E}\left[\left(\delta \theta_{0}+\Delta \widehat{\theta}_{0}\right)^{2}\right]=\left[\Delta \widehat{\theta}_{0}\right]^{2}+\mathbb{E}\left[\delta \theta_{0}^{2}\left(\widehat{\Theta}_{0}, f\right)\right]$
$\operatorname{MSE}_{\phi}\left(f, \phi_{0}\right)=\mathbb{E}\left[\left(\delta \phi_{0}+\Delta \widehat{\phi}_{0}\right)^{2}\right]=\left[\Delta \widehat{\phi}_{0}\right]^{2}+\mathbb{E}\left[\delta \phi_{0}^{2}\left(\widehat{\Theta}_{0}, f\right)\right]$

with

$$
\left[\Delta \widehat{\phi}_{0}\right]^{2}=0, \quad\left[\Delta \widehat{\theta}_{0}\right]^{2}=\left(\sin ^{-1}\left(\frac{f_{0}}{f} \sin \left(\theta_{0}\right)\right)-\theta_{0}\right)^{2} .
$$

In Appendix $\mathrm{A}$ it is shown that $\mathbb{E}\left[\delta \theta_{0}\right]=\mathbb{E}\left[\delta \phi_{0}\right]=0$, and in Appendix B explicit expressions for $\mathbb{E}\left[\delta \theta_{0}^{2}\left(\widehat{\Theta}_{0}, f\right)\right]$ and $\mathbb{E}\left[\delta \phi_{0}^{2}\left(\widehat{\Theta}_{0}, f\right)\right]$ are derived and expressed by (58).
Given the transmitted signal power spectral density (PSD) $P_{\mathrm{t}}(f)$, with bandwidth $W$, we finally define the pointing root mean square error (PRMSE) as

$$
\operatorname{PRMSE}\left(\Theta_{0}\right)=\sqrt{\frac{\int_{W} P_{\mathrm{t}}(f)\left[\mathrm{MSE}_{\theta}\left(f, \theta_{0}\right)+\mathrm{MSE}_{\phi}\left(f, \phi_{0}\right)\right] d f}{\int_{W} P_{\mathrm{t}}(f) d f}} .
$$

It will be shown in Sec. 5 that small bandwidths $W$ are better suited for high beam pointing accuracy. This is in contrast with the need to have large bandwidth signals for accurate ranging and a trade-off has to be found as will be reported in the numerical results.

\subsection{Beamwidth Spread}

We are now interested in finding the $3 \mathrm{~dB}$ beamwidth spread $\widetilde{\Theta}_{3 \mathrm{~dB}}=\left(\tilde{\theta}_{0} \pm \delta \theta, \tilde{\phi}_{0} \pm \delta \phi\right)$, which varies with the frequency even if this is not explicitly indicated for notation convenience. In particular, the half power beamwidth (HPBW) can be evaluated by solving the following equation

$$
\left|\operatorname{AF}\left(\widetilde{\Theta}_{3 \mathrm{~dB}}, f\right)\right|^{2}=\frac{1}{2}\left|\operatorname{AF}\left(\widetilde{\Theta}_{0}, f\right)\right|^{2} .
$$

It follows that

$$
\sum_{m n p q} \hat{w}_{m n p q} e^{j(m-p) \Psi_{\delta_{x}}} e^{j(n-q) \Psi_{\delta_{y}}} e^{j\left(\delta_{m n}-\delta_{p q}\right)}=\frac{1}{2}\left|\operatorname{AF}\left(\widetilde{\Theta}_{0}, f\right)\right|^{2}
$$

where $\hat{w}_{m n p q}=e^{j\left[\left.(m-p) \Psi_{x}\right|_{\Theta=\tilde{\Theta}_{0}}+\left.(n-q) \Psi_{y}\right|_{\Theta=\tilde{\Theta}_{0}}\right]}$ and

$$
\begin{aligned}
& \Psi_{\delta \mathrm{x}}=k d_{\mathrm{x}}\left[\cos \left(\tilde{\theta}_{0}\right) \cos \left(\tilde{\phi}_{0}\right) \delta \theta-\sin \left(\tilde{\theta}_{0}\right) \sin \left(\tilde{\phi}_{0}\right) \delta \phi\right] \\
& \Psi_{\delta \mathrm{y}}=k d_{\mathrm{y}}\left[\sin \left(\tilde{\theta}_{0}\right) \cos \left(\tilde{\phi}_{0}\right) \delta \phi+\cos \left(\tilde{\theta}_{0}\right) \sin \left(\tilde{\phi}_{0}\right) \delta \theta\right] .
\end{aligned}
$$

In Appendix $C$ a practical expression for the numerical evaluation of (13) is derived. From (62) and (63) in Appendix $\mathrm{C}$ we introduce the beam solid area (BSA)

$$
\mathrm{BSA}=\Omega_{\mathrm{A}}\left(\Theta_{0}, f\right)=4 \mathbb{E}\left[\delta \tilde{\theta}_{x 0}\left(\widetilde{\Theta}_{0}, f\right)\right] \mathbb{E}\left[\delta \tilde{\theta}_{y 0}\left(\widetilde{\Theta}_{0}, f\right)\right] \sec \tilde{\theta}_{0} .
$$

In addition, we define the effective $B S A$ as

$$
\Omega_{\mathrm{A}, \text { eff }}\left(\Theta_{0}\right)=\sqrt{\frac{\int_{W} P_{\mathrm{t}}(f) \Omega_{\mathrm{A}}^{2}\left(\Theta_{0}, f\right) d f}{\int_{W} P_{\mathrm{t}}(f) d f}} .
$$

This parameter is an indicator of the angle resolution of the scanning process at direction $\Theta_{0}$.

It will be shown in Sec. 5 through simulations that a large number of elements lets to reduce the impact of quantization errors as well as to narrow the beamwidth.s 


\subsection{Ranging and Steering Accuracy}

We now characterize the ranging and angle resolution of massive antenna arrays accounting for bandwidth and sources of errors discussed in Sec. 2.

Target distance estimate (ranging) during each step of the scanning process is obtained through the measurement of the signal round-trip time (RTT). The fundamental limit of ranging accuracy of a target at distance $d$ depends on the signal effective bandwidth $W_{\text {eff }}$ and the received signal-to-noise ratio (SNR) as given by the Cramér-Rao lower bound (CRLB) [31]

$$
\mathrm{CRLB}=\frac{c^{2}}{8 \pi^{2} \mathrm{SNR} W_{\mathrm{eff}}^{2}}
$$

where $W_{\text {eff }}$ is defined as

$$
W_{\text {eff }} \triangleq \sqrt{\frac{\int_{-\infty}^{+\infty} f^{2} P_{\mathrm{t}}\left(f-f_{0}\right) d f}{\int_{-\infty}^{+\infty} P_{\mathrm{t}}\left(f-f_{0}\right) d f}} \approx W .
$$

The $\mathrm{mmW}$ radar range equation related to a target at distance $d$, a test steering direction $\Theta_{0}$, and considering a waveform with incident direction $\Theta_{i}=\left(\theta_{i}, \phi_{i}\right)$ with respect to the target, can be expressed as

$P_{\mathrm{r}}\left(\Theta_{0}, \Theta_{i}, d\right)=\int_{W} \frac{P_{\mathrm{t}}(f) c^{2} G^{2}\left(\Theta_{0}, f\right) M\left(\Theta_{0}, \Theta_{i}, f, d\right)}{f^{2}(4 \pi)^{3} d^{4}} d f$

with $G\left(\Theta_{0}, f\right) \approx \pi^{2} / \Omega_{\mathrm{A}}\left(\Theta_{0}, f\right)$, being the array gain [29] and $M\left(\Theta_{0}, \Theta_{i}, f, d\right)$ the target radar cross section (RCS).

Under the narrow beamwidth hypothesis, i.e. high $N_{\text {array }}$, we approximate the illuminated area on the target surface with the beam area of the incident wave generated by the massive array (footprint area). Thus, the RCS of a target at $\mathrm{mmW}$ is given by [33], [34]

$$
M\left(\Theta_{0}, \Theta_{i}, f, d\right)=4 \pi \rho A_{\mathrm{fp}}\left(\Theta_{0}, f, d\right) \zeta\left(\Theta_{i}\right)
$$

where $\rho$ is a parameter which depends on the type of scattering and the target material, and $\zeta\left(\Theta_{i}\right)$ is the incident/reflection coefficient of the target in the direction $\Theta_{i}$. The footprint area is

$$
A_{\mathrm{fp}}(\Theta, f, d)=\frac{\pi}{4} d^{2} \Omega_{\mathrm{A}}(\Theta, d)
$$

with $\Omega_{\mathrm{A}}(\Theta, f)$ given by (16). Thus (20) results

$$
P_{\mathrm{r}}\left(\Theta_{0}, \Theta_{i}, d\right)=\frac{\pi c^{2}}{64} \int_{W} \frac{\operatorname{EIRP}(f) \rho \zeta\left(\Theta_{i}\right)}{d^{2} f^{2}} d f
$$

where $\operatorname{EIRP}(f)=P_{\mathrm{t}}(f) G\left(\Theta_{0}, f\right)$ is the effective radiated isotropic power (EIRP) typically constrained by regulatory power emission limits. Note the quadratic dependency of $P_{\mathrm{r}}\left(\Theta_{0}, \Theta_{i}, d\right)$ on the mobile-target distance $d$.

The actual SNR can be improved by increasing the transmission power or the observation time (e.g., by transmitting several pulses/symbols). However, both parameters have some constraints on spectrum emission regulations and the maximum tolerable scanning time, respectively. For what the latter is regarded, suppose we have to scan half of the full solid angle or half of the full angle, i.e. $\Omega_{\mathrm{o}}=2 \pi[\mathrm{sr}]$ and $\Omega_{\mathrm{o}}=\pi[\mathrm{rad}]$ in $3 \mathrm{D}$ and
2D scenarios, respectively. The number $N_{\text {steer }}$ of steering test directions to complete the scan process is strictly related to the array beamwidth, and thus to the number of antenna elements $N_{\text {array }}$, by the relation ${ }^{2}$

$$
N_{\text {steer }}=\left\lfloor\frac{\Omega_{\mathrm{o}}}{\Omega_{\text {mean }}}\right\rfloor
$$

where $\Omega_{\text {mean }}$ denotes the mean BSA HPBW averaged over all the test directions $\Theta_{0}$ given by

$$
\Omega_{\text {mean }}=\frac{1}{\Omega_{\mathrm{O}}} \int_{\Omega_{\mathrm{o}}} \Omega_{\mathrm{A}, \mathrm{eff}}(\Theta) d \Omega .
$$

Note that $\Omega_{\text {mean }}$ provides an indication on the scanning average angle resolution.

As a consequence, the scanning time is $T_{\text {scan }}=$ $T_{\text {ob }} N_{\text {steer, }}$ with $T_{\text {ob }}$ representing the observation time for each test direction. Note the dependency on the BSA: a narrow beam increases the scanning time, while the scanning angle resolution is improved. Under scanning time constraints, the SNR is given by

$$
\mathrm{SNR}=\frac{P_{\mathrm{r}}\left(\Theta_{0}, \Theta_{i}, d\right) T_{\mathrm{ob}}}{N_{0}}=\frac{P_{\mathrm{r}}\left(\Theta_{0}, \Theta_{i}, d\right) T_{\mathrm{scan}}}{N_{\text {steer }} N_{0}}
$$

where $N_{0}=N_{\text {array }} \kappa \cdot T_{0} \cdot F$, being $\kappa$ the Boltzmann constant, $T_{0}=290[\mathrm{~K}]$ the receiver temperature, and $F$ the receiver noise figure. Eq. (26) can be used in (18) to derive the fundamental limit of ranging as a function of the average BSA and scanning time constraint as it will be investigated in the numerical results.

\section{Personal Radar Mapping Capability}

The previous analysis has highlighted how angle resolution, scanning time, signal bandwidth and ranging accuracy are tightly coupled.

We investigate now the mapping capabilities of the personal radar integrating massive arrays working at mmW. We consider a person with a mobile terminal (e.g., a smartphone or a tablet) equipped with a $\mathrm{mmW}$ massive antenna array who walks with constant speed along a pre-determined trajectory and whose position and orientation are constantly determined and updated by sensors integrated on the terminal or by external localization technologies [35]. The main task of personal radar is to incrementally build a consistent $2 \mathrm{D} / 3 \mathrm{D}$ map of the surrounding environment. To this purpose, first we propose a $\mathrm{mmW}$ radar collecting a rich set of measurements from the massive antenna array. Then a grid-based probabilistic mapping approach is adopted to fully exploit such measurements in a static environment. Hereafter, for simplicity of notation, we will restrict our analysis to a $2 \mathrm{D}$ scenario even if the $3 \mathrm{D}$ extension is straightforward.

2. $\lfloor x\rfloor$ denotes the biggest integer smaller than $x$. 


\subsection{Massive Antenna Array Radar}

The purpose of the radar is to provide a rich set of observations for the subsequent mapping phase starting from the analysis of the signals backscattered by the surrounding environment.

Let $\theta_{b}=-\pi / 2\left(\frac{N_{\text {steer }}-1}{N_{\text {steer }}}\right)+(b-1) \pi / N_{\text {steer, }} b=$ $1,2, \ldots N_{\text {steer, }}$ be the set of $N_{\text {steer }}$ steering directions considered during the scanning process defined, and $\theta_{v}$ the mobile orientation.

Consider a generic interrogation signal, for each steering direction $\theta_{b}$, composed of $N_{\mathrm{p}}$ wideband pulses $p(t)$

$$
g(t)=\sum_{i=0}^{N_{\mathrm{p}}-1} p\left(t-i T_{\mathrm{f}}\right)
$$

with $T_{\mathrm{f}}$ being the time frame chosen so that all signals backscattered by the environment are received by the receiver before the transmission of the successive pulse, thus avoiding inter-frame interference. The consequent scanning time is $T_{\text {scan }}=T_{\mathrm{ob}} N_{\text {steer, }}$, where $T_{\mathrm{ob}}=N_{\mathrm{p}} T_{\mathrm{f}}$.

Since each pulse is backscattered by the surrounding targets populating the environment, for the steering direction $\theta_{b}$ the received signal can be expressed as

$$
r\left(t, \theta_{b}\right)=\sum_{i=0}^{N_{\mathrm{p}}-1} s\left(t-i T_{\mathrm{f}}, \theta_{b}\right)+n(t)
$$

where $s\left(t, \theta_{b}\right)=p(t) \otimes h_{\mathrm{C}}\left(t, \theta_{b}\right)$ is the channel response to the transmitted pulse $p(t)$ at direction $\theta_{b}$, with $h_{\mathrm{C}}\left(t, \theta_{b}\right)$ being the channel impulse response (CIR), de-embedded of the propagation time, taking the joint environment and antenna array response into account, ${ }^{3}$ and with $n(t)$ being the additive white Gaussian noise (AWGN) with two-sided power spectral density $N_{0} / 2$.

The received signal is first passed through an ideal bandpass filter with center frequency $f_{0}$ to eliminate outof-band noise. ${ }^{4}$ The filtered signal is denoted by

$$
y\left(t, \theta_{b}\right)=\sum_{i=0}^{N_{\mathrm{p}}-1} x\left(t-i T_{\mathrm{f}}, \theta_{b}\right)+z(t)
$$

where $x\left(t, \theta_{b}\right)=s\left(t, \theta_{b}\right) \otimes h_{\mathrm{F}}(t) \cong s\left(t, \theta_{b}\right)$ and $z(t)=n(t) \otimes$ $h_{\mathrm{F}}(t)$, with $h_{\mathrm{F}}(t)$ being the impulse response of the filter.

To conjugate the need of having a manageable number of measurements and a low complexity receiver, we consider a non-coherent approach based on energy measurements in a discretized time scale. This approach also accounts for the complete uncertainty on the received waveform shape deriving from the a priori ignorance on environment electromagnetic characteristics. Specifically, energy measurements are taken during the time frame $T_{\mathrm{f}}$ after the transmission of each pulse by subdividing the time frame into $N_{\text {bin }}=\left\lfloor T_{\mathrm{f}} / T_{\mathrm{ED}}\right\rfloor$ time slots (bins) of duration $T_{\mathrm{ED}}$. Energy measurements are accumulated for each time bin over the $N_{\mathrm{p}}$ frames of the interrogation

3. $\otimes$ is the convolutional operator

4. This operation is necessary since the receiver is energy-based, as it will be described later. signal. The accumulated energy measurement at the $s$ th time bin and $b$ th steering angle $\theta_{b}$ is

$$
e_{b s}=\sum_{k=0}^{N_{\mathrm{p}}-1} \int_{(s-1) T_{\mathrm{ED}}}^{s T_{\mathrm{ED}}} y^{2}\left(t+k T_{\mathrm{f}}, \theta_{b}\right) d t
$$

with $s=1,2, \ldots, N_{\text {bin }}$ and $b=1,2, \ldots N_{\text {steer. Note that }}$ $T_{\mathrm{ED}}$ must be chosen to accommodate most of the energy of the received pulse, i.e. $T_{\mathrm{ED}} \approx 1 / W$.

According to [36], for each energy bin, the normalized energy measurement output can be well approximated by

$$
\Lambda_{b s}=\frac{2}{N_{0}} e_{b s} \simeq \frac{1}{\sigma^{2}} \sum_{k=0}^{N_{\mathrm{p}}-1} \sum_{i=(s-1) N_{\mathrm{d}}}^{s N_{\mathrm{d}}}\left(x_{i}\left(\theta_{b}\right)+z_{i}^{(k)}\right)^{2}
$$

where $N_{\mathrm{d}}=2 W T_{\mathrm{ED}}, \sigma^{2}=N_{0} W$ is the noise variance, and $z_{i}^{(k)}$ are for odd $i$ (even $i$ ) the samples of the real (imaginary) part of the equivalent low-pass of $z\left(t+k T_{\mathrm{f}}\right)$, $k=1,2, \ldots, N_{\mathrm{p}}$, taken at Nyquist rate $W$ in each interval $T_{\mathrm{ED}}$. In (31) we used the property $x\left(t+k T_{\mathrm{f}}, \theta_{b}\right)=x\left(t, \theta_{b}\right)$, with $k=1,2, \ldots, N_{\mathrm{p}}$, so that $x_{i}\left(\theta_{b}\right)$ represents for odd $i$ (even $i$ ) the samples of the real (imaginary) part of the equivalent low-pass of $x\left(t, \theta_{b}\right)$, taken at Nyquist rate $W$ in each interval $T_{\mathrm{ED}}$, which does not depend on $k$.

It turns out that $\Lambda_{b s}$ is a non-central Chi-square distributed RV with $N=N_{\mathrm{p}} N_{\mathrm{d}}$ degrees of freedom. The non-centrality parameter is $\lambda_{b s}=2 \gamma_{b s}$ [36], where $\gamma_{b s}$ is given by

$$
\gamma_{b s}=\frac{N_{\mathrm{p}}}{2 \sigma^{2}} \sum_{i=(s-1) N_{\mathrm{d}}}^{s N_{\mathrm{d}}} x_{i}^{2}\left(\theta_{b}\right) \simeq \frac{N_{\mathrm{p}}}{N_{0}} \int_{(s-1)}^{s T_{\mathrm{ED}}} x_{T_{\mathrm{ED}}}^{2}\left(t, \theta_{b}\right) d t .
$$

Note that (32) represents the accumulated SNR corresponding to the $s$ th time bin and the $b$ th steering direction which increases with the number $N_{\mathrm{p}}$ of pulses at the expense of a longer observation time $T_{\mathrm{ob}}$. Furthermore, each energy measurement $e_{b s}$ does not depend only on the signal backscattered in the steering direction $\theta_{b}$, but also on all contributions coming from different directions according to the shape of the array radiation pattern which affects $h_{\mathrm{C}}\left(t, \theta_{b}\right)$ and hence $x\left(t, \theta_{b}\right)$. During the mapping process these contributions could create ambiguities but, at the same time, permit to discriminate between different array configurations.

\subsection{Probabilistic State-Space Model for Mapping}

In the following, we introduce a state-space model for probabilistic mapping able to exploit the measurements from the massive array radar under a Bayesian filtering approach [18].

In a probabilistic mapping framework, the position and orientation of the mobile is known or partially known if resulting from an external localization system exploiting, for example, inertial measurement unit (IMU) and RF sensors measurements. The same mmW technology could be in principle exploited to provide 


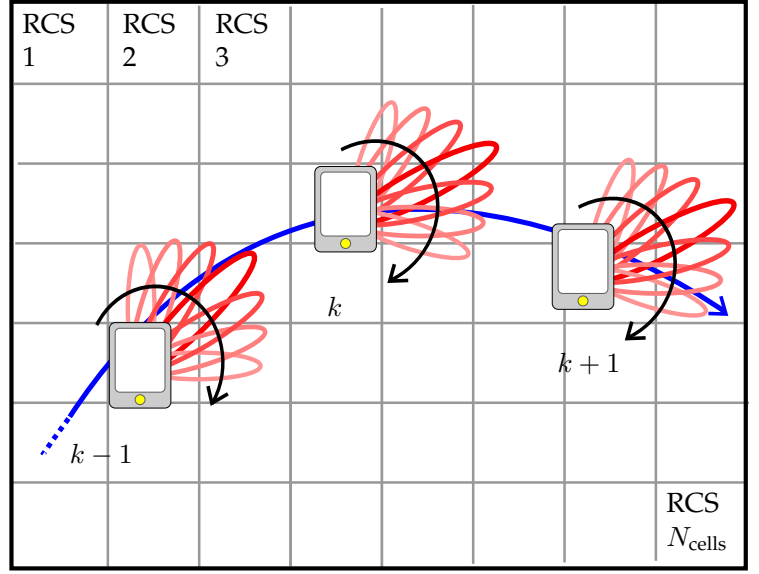

Fig. 3: Grid-based mapping performed with the personal radar in three different positions at the instants $k-1, k$ and $k+1$.

extremely high accuracy positioning down to a few $\mathrm{cm}$ when interacting with base stations [26].

Different models can be adopted to represent the map. Feature-based models are privileged when a set of landmarks characterizes the environment and the sensors on the mobile device are able to well-discriminate them (i.e. using laser and video technologies). On the contrary, grid-based models for map representation are more suitable when sensors provide imprecise measurements from which it is difficult to discriminate between different features, such as when using radar [37]. In gridbased approaches, the environment is discretized in cells whose position is a-priori known and hence the number of states is fixed to the number of cells. In most of works, the state is a binary RV and represents the presence or not of any physical element in the corresponding cell (occupancy grid) [38].

In our work we consider RCS-based description of cells in a map-grid representation context, and the adoption of massive array radars with electronically steering beams instead of mechanically steering devices. In particular, a 2D grid of $N_{\mathrm{L}}=X_{\text {grid }} Y_{\text {grid }}$ cells representative of the environment is considered. Differently from previous works, we propose a one-step procedure where a larger observation vector is introduced accounting for the specific massive antenna array radiation pattern, investigated in Secs. 2 and 3, and whose response could depend, in principle, on several cells, as illustrated in Fig. 3. Therefore, the mapping process works with a richer information set and no specific radar detection scheme and associated detection threshold evaluation strategy have to be designed, as required instead in conventional two-step approaches [15]. In the following, the adopted scheme is described, and the differences with classic Bayesian SLAM are highlighted.

\subsubsection{State Vector}

We define the state vector at time $k$ as

$$
\mathbf{x}(k)=\mathbf{m}(k)=\left[m_{1}(k), \ldots, m_{i}(k), \ldots, m_{N_{\mathrm{L}}}(k)\right]^{\mathrm{T}}
$$

where $m_{i}(k)$ indicates the root radar cross section (RRCS) (with sign) of the $i$ th cell of the grid. Obviously, if the cell is empty (only air), the corresponding RRCS is zero. Note that in the model herein adopted we neglect the dependency of $m_{i}(k)$ from the frequency and the environment is assumed stationary.

\subsubsection{Observation Model}

Define $\mathbf{e}(k)$ the vector containing the accumulated measured energy at the output of the receiver at time $k$

$$
\begin{aligned}
\mathbf{e}(k)= & {\left[e_{11}(k), \ldots, e_{1 N_{\text {bin }}}(k), \ldots, e_{b s}(k), \ldots,\right.} \\
& \left.e_{N_{\text {steer }} 1}(k), \ldots e_{N_{\text {steer }} N_{\text {bin }}}(k)\right]^{\mathrm{T}}
\end{aligned}
$$

where $e_{b s}(k)$ is given by (30). As shown in the previous section, $e_{b s}(k)$ is proportional to a non-central Chisquare distributed RV that makes the formulation of the observation model challenging. However, for large $N$ (typically $>50$ ), i.e. for large $N_{\mathrm{p}}$, the non-central Chi-square distribution can be approximated with a Gaussian distribution having mean $\lambda_{b s}+N$ and variance $2\left(N+2 \lambda_{b s}\right)$ [39]. We exploit this property to construct a tractable Gaussian observation model of the state-space model

$$
\begin{aligned}
\mathbf{z}(k) & =\left[z_{11}(k), \ldots, z_{b s}(k), \ldots, z_{N_{\text {steer }} N_{\text {bin }}}(k)\right]^{\mathrm{T}} \\
& =\mathbf{h}(\mathbf{x}(k))+\mathbf{v}(k)
\end{aligned}
$$

where $z_{b s}(k)$ is the energy observation corresponding to the $s$ th time bin and the $b$ th steering angle, $\mathbf{h}(\cdot)$ is the observation function that relates the state vector to the observations, and

$$
\mathbf{v}(k)=\left[v_{11}(k), \ldots, v_{b s}(k), \ldots, v_{N_{\text {steer }} N_{\text {bin }}}(k)\right]^{\mathrm{T}}
$$

is the vector of uncorrelated Gaussian observation errors with zero mean and covariance matrix $\mathbf{R}(k)=$ $\operatorname{diag}\left(\sigma_{11}^{2}(k), \ldots, \sigma_{b s}^{2}(k), \ldots, \sigma_{N_{\text {steer }} N_{\text {bin }}}^{2}(k)\right)$ with $\sigma_{b s}^{2}(k)=$ $\operatorname{Var}\left(e_{b s}(k)\right)$. Note that the length $N_{\mathrm{m}}=N_{\text {steer }} N_{\text {bin }}$ of $\mathbf{z}(k)$ is related either to the number of the steering directions, depending on the antenna array pattern $G(\theta, f)$, and to the number of time bins depending on the signal bandwidth $W$ through $T_{\mathrm{ED}}$.

The generic element of $\mathbf{z}(k)$ can be written as

$$
z_{b s}(k)=h_{b s}(\mathbf{x}(k))+v_{b s}(k)=\mathbb{E}\left[e_{b s}(k)\right]+v_{b s}(k)
$$

with $b=1,2, \ldots, N_{\text {steer, }} s=1,2, \ldots, N_{\text {bin }}$.

From (31) and (32) it is $\mathbb{E}\left[e_{b s}(k)\right]=T_{\mathrm{f}} P_{b s}(k)$ whereas $v_{b s}(k)$ is the measurement noise, as defined in (36), with variance $\sigma_{b s}^{2}(k)=N_{0}\left(N_{\mathrm{p}} W T_{\mathrm{ED}} N_{0}+2 T_{\mathrm{f}} P_{b s}(k)\right)$. Using (21), the term $P_{b s}(k)$ depends on $\mathbf{x}(k)$ and represents the average received power at the $b$ th steering angle and the $s$ th time bin given by

$$
\begin{aligned}
& P_{b s}(k)=\frac{\mathbb{E}\left[e_{b s}(k)\right]}{T_{\mathrm{f}}} \\
& =P_{w}+\sum_{i \in \mathcal{R}(s)} \int_{W} \frac{P_{t}(f) c^{2} m_{i}^{2}(k) G^{2}\left(\theta_{i}-\theta_{v}-\theta_{b}, f\right) N_{\mathrm{p}}}{f^{2}(4 \pi)^{3} d_{i}^{4}} d f
\end{aligned}
$$


where $P_{w}=\frac{\sigma^{2} T_{\mathrm{ED}} N_{\mathrm{p}}}{T_{\mathrm{P}}}$ is the noise power, $d_{i}$ and $\theta_{i}$ are, respectively, the distance and angle between the mobile and the $i$ th cell. Function $\mathcal{R}(s)$ returns the set of cells indexes contributing to the $s$ th time bin (i.e. all the cells located at a distance close to $s \cdot d_{\text {bin }}$ with $\left.d_{\text {bin }}=c \cdot T_{\mathrm{ED}} / 2\right)$.

Note that in (38) the actual footprint area corresponds to the aggregate area of the cells illuminated by the beam. In fact, $m_{i}(k)$ is evaluated using (21), by setting the footprint area $A_{\mathrm{fp}}$ to the area of a cell.

\subsection{EKF-Mapping}

There are several recursive approaches belonging to the Bayesian filtering theory to evaluate the posterior distribution $p(\mathbf{x}(k) \mid \mathbf{z}(1: k))$ of $\mathbf{x}(k)$ given the set of measurements $\mathbf{z}(1: k)=\{\mathbf{z}(1), \mathbf{z}(2), \ldots, \mathbf{z}(k)\}$ collected by the mobile device until time instant $k$. Among them, here we employ the extended Kalman-Filter (EKF) which offers a good trade-off between performance and complexity considering the intrinsic large state vector of dimension $N_{\mathrm{L}}$ resulting from the grid-based map representation.

The mean and covariance state matrix of the predicted state are, in linear, $\hat{\mathbf{x}}(k+1 \mid k)=\hat{\mathbf{x}}(k \mid k)$ and $\mathbf{P}(k+1 \mid k)=\mathbf{P}(k \mid k)$ respectively, due to the stationarity of the environment. ${ }^{5}$ The prediction for the observation model is given by $\hat{\mathbf{z}}(k+1 \mid k)=\mathbf{h}(\hat{\mathbf{x}}(k+1 \mid k))$. Once the new measurement vector $\mathbf{e}(k+1)$ is available, the innovation in the model is

$$
\boldsymbol{\nu}(k+1)=\mathbf{e}(k+1)-\hat{\mathbf{z}}(k+1 \mid k) .
$$

Consequently, the correction step performed by the EKF is described by

$$
\begin{aligned}
& \hat{\mathbf{x}}(k+1 \mid k+1)=\hat{\mathbf{x}}(k+1 \mid k)+\mathbf{W}(k+1) \boldsymbol{\nu}(k+1) \\
& \mathbf{P}(k+1 \mid k+1)=\mathbf{P}(k+1 \mid k)-\mathbf{W}(k+1) \mathbf{S}(k+1) \mathbf{W}^{\mathrm{T}}(k+1)
\end{aligned}
$$

where

$$
\begin{aligned}
& \mathbf{S}(k+1)=\nabla \mathbf{h} \mathbf{P}(k+1 \mid k) \nabla \mathbf{h}^{T}+\mathbf{R}(k+1) \\
& \mathbf{W}(k+1)=\mathbf{P}(k+1 \mid k) \nabla \mathbf{h}^{T} \mathbf{S}^{-1}(k+1)
\end{aligned}
$$

with $\nabla \mathbf{h}$ being the Jacobian of $\mathbf{h}(\cdot)$ evaluated in $\hat{\mathbf{x}}(k+$ $1 \mid k)$, which gives using (37) and (38)

$\frac{\partial h_{b s}}{\partial m_{i}}= \begin{cases}2 m_{i} \int_{W} \frac{P_{t}(f) c^{2} G^{2}\left(\theta_{i}-\theta_{\mathrm{v}}-\theta_{b}, f\right) T_{\mathrm{f}} N_{\mathrm{p}}}{f^{2}(4 \pi)^{3} d_{i}^{4}} d f & \text { if } i \in \mathcal{R}(s) \\ 0 & \text { otherwise. }\end{cases}$

In the numerical results a case study is provided implementing the state-space model and the EKF mapping approach here introduced.

\section{Numerical Results}

We first evaluate the impact of frequency selectivity and quantization errors on antenna array characteristics in order to investigate the ranging and steering accuracy as a function of $N_{\text {array }}$. Successively, mapping capability is analyzed by exploiting the new state-space model proposed in Sec. 4.

5. The notation $(m \mid n)$ indicates at time $m$ given the observations until time $n$.

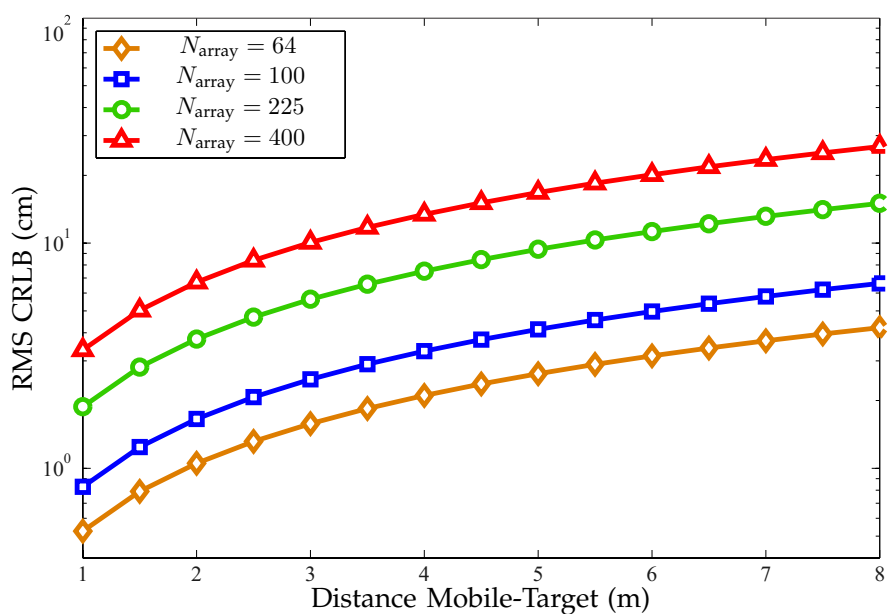

Fig. 4: RMS CRLB vs. mobile-target distance for different values of $N_{\text {array }}, \Theta_{0}=\left(45^{\circ}, 45^{\circ}\right)$, and $W=500 \mathrm{MHz}$.

\subsection{System Parameters}

According to the wavelength $\lambda_{0}$ and the object size $L$, there are different scattering regimes. Coherently with [34], we assume here to have $\lambda_{0} \ll L$ (optics regime) even if in some cases it could result in a rough approximation. This assumption is reasonable in indoor environments where most of targets are constituted by walls that have an extension much larger than the wavelength. We consider the approximation of a Lambertian scattering [40], adopting the laser model proposed in [33] for the monostatic laser RCS, where $\rho$ is defined as $\rho=\rho_{\mathrm{d}} / \pi$, with $\rho_{\mathrm{d}}$ being the diffuse reflectivity (albedo), and $\Theta_{i}$ normal to the target. In addition, we assume a target made of aerate concrete by approximating the albedo with the power reflection coefficient, evaluated by setting the concrete relative permittivity to $\epsilon_{r}=2.26$ and its loss tangent to 0.0491 [41]. We also considered a scanning time $T_{\text {scan }}=0.1 \mathrm{~ms}, F=4 \mathrm{~dB}$ and the transmission of a root raised cosine (RRC) signal compliant with the Federal Communications Commission (FCC) mask at $60 \mathrm{GHz}$ [42], but with an average EIRP limited to $30 \mathrm{dBm}$ to make the system suitable for battery-powered handset devices.

\subsection{Ranging and Steering Accuracy Trade-off}

In this section, an example of trade-off between ranging and angle resolution is given considering a planar array working at $60 \mathrm{GHz}$ with $d_{\mathrm{x}}=d_{\mathrm{y}}=\lambda_{0} / 2$ and $M=N$.

The RMS CRLB on ranging is reported in Fig. 4, obtained for different mobile-target distances and for different values of array elements $N_{\text {array, }}$ where the transmitted signal bandwidth is set to $W=500 \mathrm{MHz}$. When $N_{\text {array }}$ increases, the SNR decreases due to the reduced BSA and the constraint on EIRP according to (26). ${ }^{6}$ In fact, $N_{\text {array }}$ enters into $N_{0}$ and the array gain, which is inversely proportional to $\Omega_{\mathrm{A}}(\Theta, f)$. Thus, large

6. Note that here performance is strictly related to the adopted model, which is valid only for a high number of antenna elements, which justify the performance degradation when $N_{\text {array }}$ increases. 


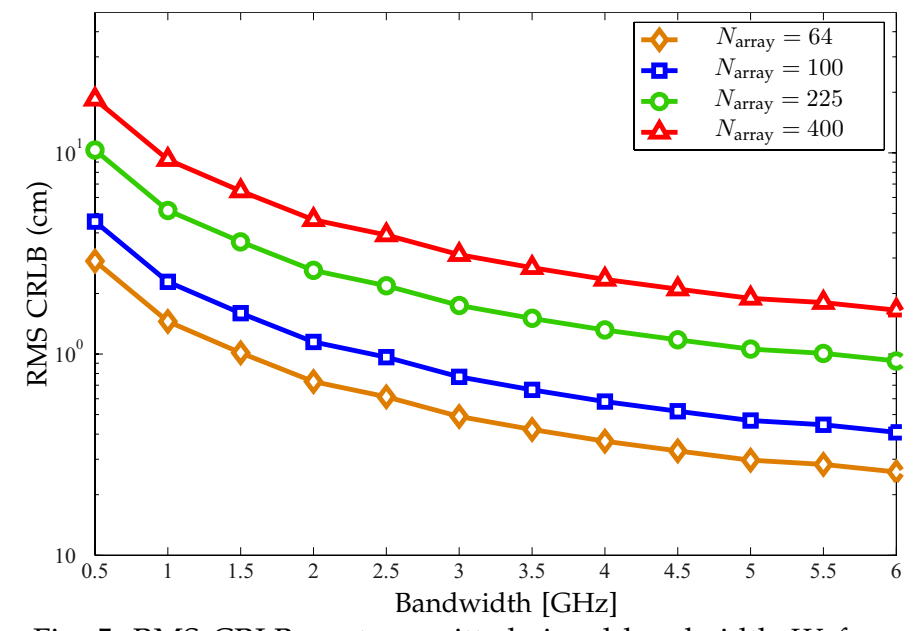

Fig. 5: RMS CRLB vs. transmitted signal bandwidth $W$ for a mobile-target distance of $10 \mathrm{~m}$, and for $\Theta_{0}=\left(45^{\circ}, 45^{\circ}\right)$.

values of $N_{\text {array }}$ improve the scanning angle resolution, but worsen the SNR affecting (18). As an example, if we set a target ranging error of $5 \mathrm{~cm}$ at $5 \mathrm{~m}$ distance, from Fig. 4 it must be $N_{\text {array }} \leq 100$. On the contrary, the bandwidth improves the ranging accuracy, as shown in Fig. 5, but it has a detrimental effect on the PRMSE, as can be noticed in Fig. 6. From the same figure it can be seen that PRMSE is quite sensitive to the test steering elevation angle $\theta_{0}$ and to phase quantization errors.

Finally, Fig. 7 reports the BSA, evaluated according to (62) and (63), whose expectation is computed through Monte Carlo simulation. Note the weak dependance of the BSA on the frequency, and how large $N_{\text {array }}$ results in lower BSA and lower sensitivity to quantization errors. In any case the effect of quantization errors on BSA can be neglected with good approximation. Moreover, if we set BSA $\leq 0.04$ [sr], it must be $N_{\text {array }} \geq 64$.

Thus, we assist to a strong impact of $N_{\text {array }}$ on the BSA, which affects both the SNR and the ranging accuracy, and hence it needs to be properly taken into account during the system design.

Furthermore, there is also the trade-off in the choice of the bandwidth: the ranging accuracy improves with a larger bandwidth but the array pointing error increases with $W$ due to frequency selectivity effects. The bandwidth and the phase errors slightly affect the effective BSA and hence the scanning angle resolution.

In our numerical example, $N_{\text {array }}=100$ and $W=$ $1 \mathrm{GHz}$ represent a reasonable compromise.

\subsection{Mapping Performance}

We implemented the previously described mapping algorithm in MATLAB in order to validate its theoretical performance.

The case study proposed refers to a typical indoor office environment of $20 \times 20 \mathrm{~m}^{2}$, whose layout is shown in Fig. 8, which has been discretized in a grid of cells having area $0.2 \times 0.2 \mathrm{~m}^{2}$ each, according to the high

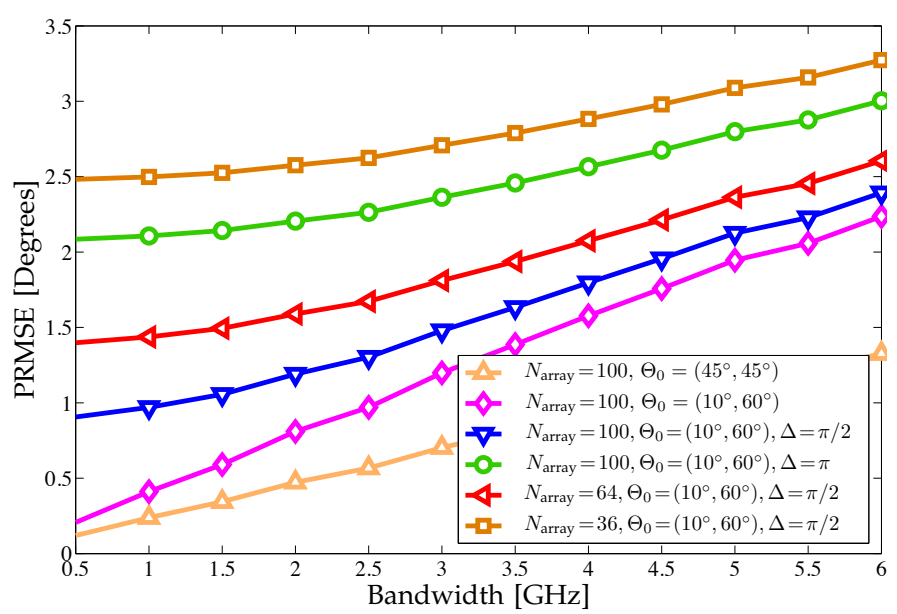

Fig. 6: PRMSE vs. bandwidth for different values of $N_{\text {array }}$, different steering angles and in presence/absence of quantization effects.

arrays resolution, and accounting for a wall attenuation, due to its thickness, of $40 \mathrm{~dB}$ [41]. The mobile device with personal radar capabilities moves along a pre-determined trajectory and speed of $1 \mathrm{~m} / \mathrm{s}$, and its reference orientation is always towards the direction of movement. Radar measurements are taken every second, and black circles refer to the true radar positions while the green squares to the positions perceived by the mobile device.

We compare the personal radar performance in three cases, $N_{\text {array }}=15 \times 15, N_{\text {array }}=10 \times 10$ and $N_{\text {array }}=4 \times 4$, respectively. In the previous numerical results, the array with $N_{\text {array }}=10 \times 10$ elements resulted as a value corresponding to a good compromise between ranging and steering accuracy. A time frame of $T_{\mathrm{f}}=100 \mathrm{~ns}$ is considered with bandwidth $W=1 \mathrm{GHz}$ and, consequently, $T_{\mathrm{ED}}=1 \mathrm{~ns}$ if not otherwise indicated. The constraint on the scanning time is fixed to $T_{\text {scan }}=80 \mu \mathrm{s}$ while the number of steering directions and of pulses per interrogation signal varies with the number of antenna elements, as $N_{\text {steer }}$ increases with $N_{\text {array }}$. In fact, for $N_{\text {array }}=16, N_{\text {array }}=100$ and $N_{\text {array }}=225$ antenna elements, it is $N_{\mathrm{p}}=100, N_{\mathrm{p}}=40$ and $N_{\mathrm{p}}=27$, respectively.

In the following results, the reconstructed map is represented in terms of RCS estimated through the EKF algorithm and it is juxtaposed to the true map of the office environment. Obviously, only the walls resulting in line-of-sight during the mobile movement are expected to be identified.

\subsubsection{Performance in Absence of System Non-Idealities}

Fig. 8 shows the estimated maps obtained with ideal (i.e. quantization error-free) antenna arrays of $4 \times 4$ (left), $10 \times 10$ (right) and $15 \times 15$ (bottom) elements, and with perfect knowledge of the mobile position and orientation. The higher number of cells that are correctly identified using the $10 \times 10$ array with respect to $4 \times 4$ and $15 \times 15$ array puts in evidence that an enhanced 


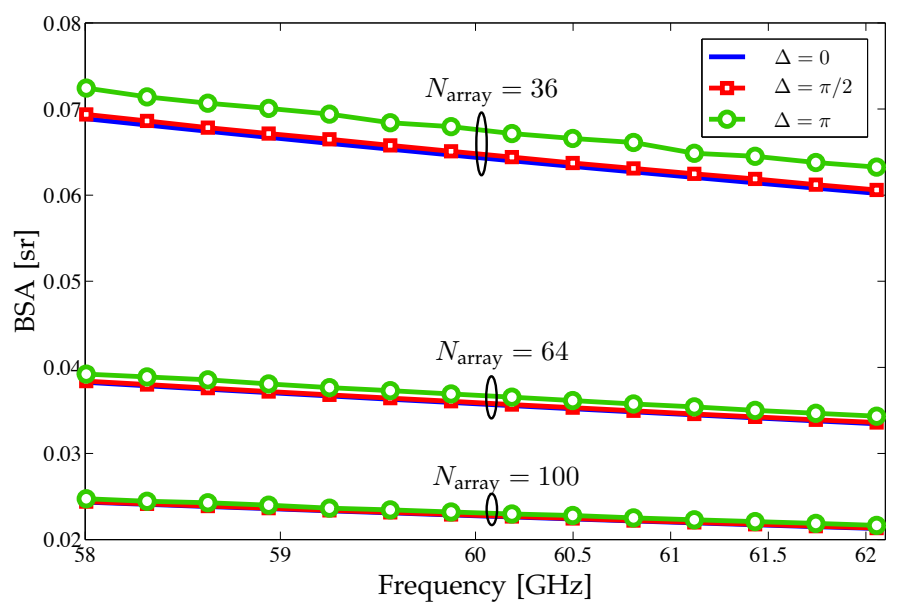

Fig. 7: BSA vs.frequency for different values of $N_{\text {array }}$ and quantization errors.
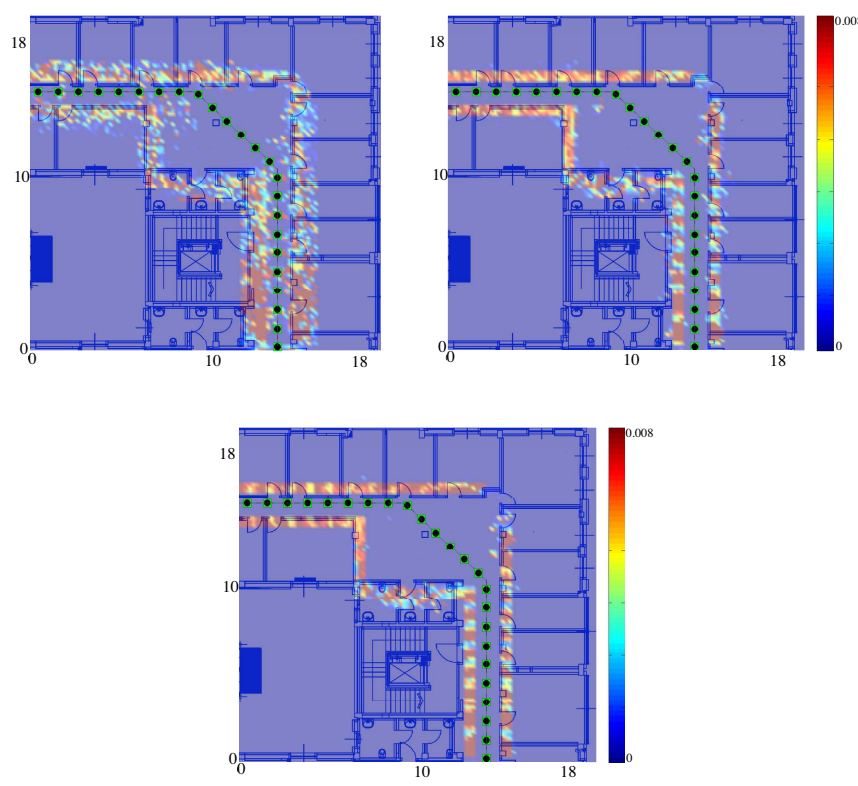

Fig. 8: 2D mapping using $4 \times 4$ (top-left), $10 \times 10$ (top-right) and $15 \times 15$ (bottom) antenna arrays with a perfect knowledge of mobile position and no quantization errors.

performance in terms of map estimation accuracy can be found as a compromise between ranging accuracy and angular resolution, in agreement with the results of Sec. 5.2. In fact, for $N_{\text {array }}=15 \times 15$, the map is well defined at the prize of a reduced SNR, and thus part of the map is not reconstructed, while for $N_{\text {array }}=4 \times 4$ the higher number of pulses improves the SNR enabling the full reconstruction of the map, but with a lower angle resolution.

Fig. 9 reports the maps estimated through the adoption of an ideal antenna array equipped with a $10 \times 10$ antenna array for different signal bandwidths. In particular, from the left to the right, the results obtained with $W=500 \mathrm{MHz}$ (i.e. $T_{\mathrm{ED}}=2 \mathrm{~ns}$ ), $W=2 \mathrm{GHz}$ (i.e. $T_{\mathrm{ED}}=0.5 \mathrm{~ns}$ ) and $W=3 \mathrm{GHz}$ (i.e. $T_{\mathrm{ED}}=0.33 \mathrm{~ns}$ ) are shown. As expected, when increasing the bandwidth the
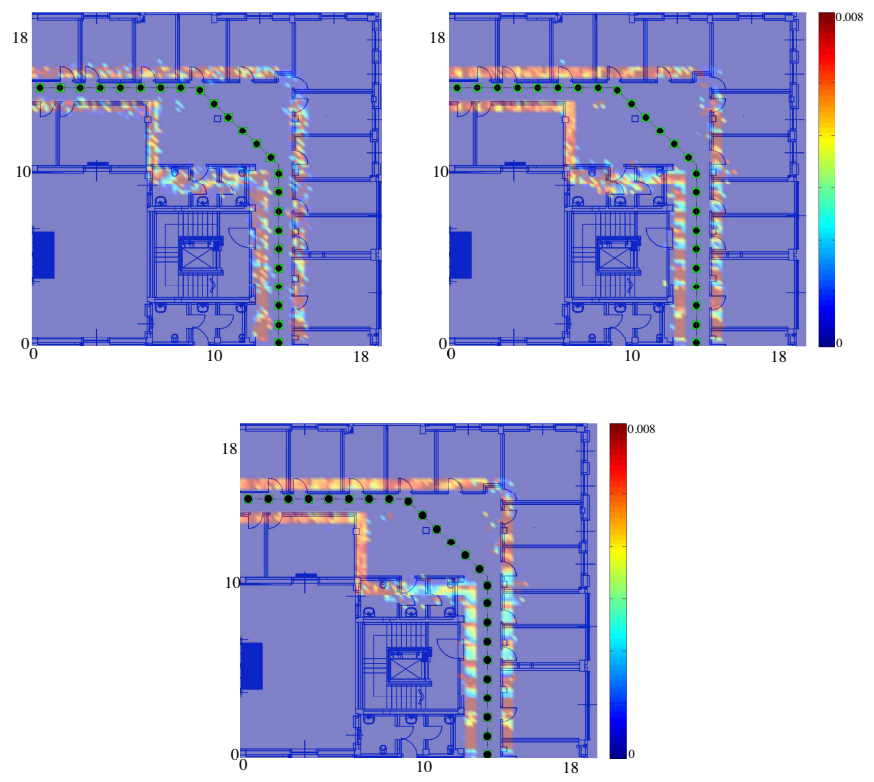

Fig. 9: 2D mapping using $10 \times 10$ antenna arrays with $W=$ $500 \mathrm{MHz}$ (top-left), $W=2 \mathrm{GHz}$ (top-right) and $W=3 \mathrm{GHz}$ (bottom) with no quantization errors and a perfect knowledge of mobile position.
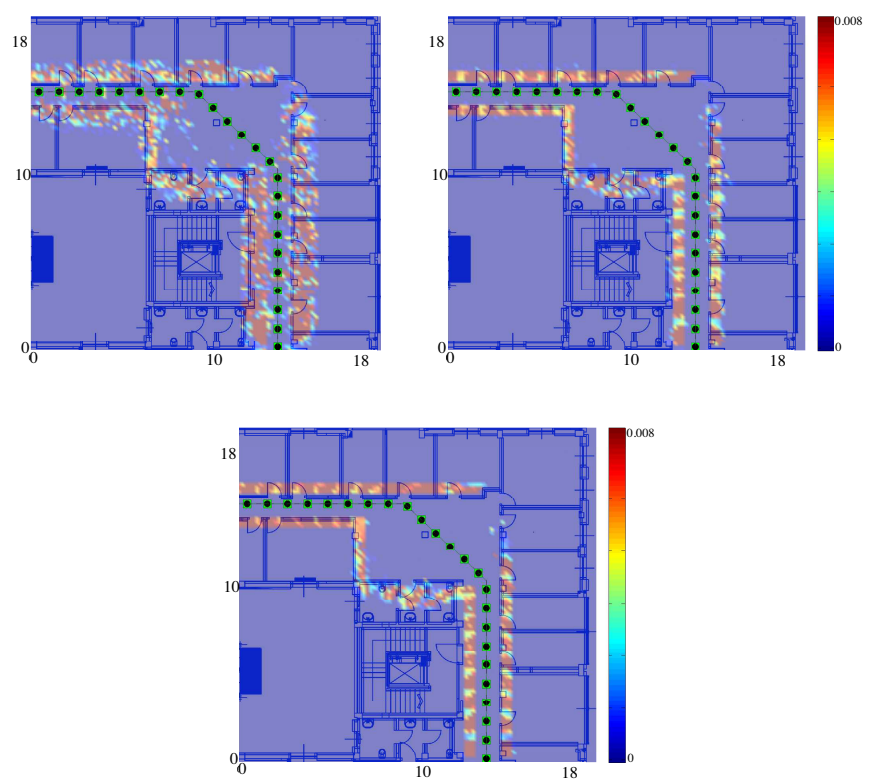

Fig. 10: 2D mapping using $4 \times 4$ (top-left), $10 \times 10$ (top-right) and $15 \times 15$ (bottom) antenna arrays with a perfect knowledge of mobile position and with array quantization errors.

mapping performance improves due to the increased time resolution at the expense of an increased number $N_{\text {bin }}$ of bins, and thus of receiver complexity.

\subsubsection{Performance with System Non-Idealities}

The effect of phase quantization errors is shown in Fig. 10 where antenna arrays with very rough phasing capability of step $\Delta=\pi / 2$ are considered. When compared to Fig. 8, it can be noted that, even if quantization 

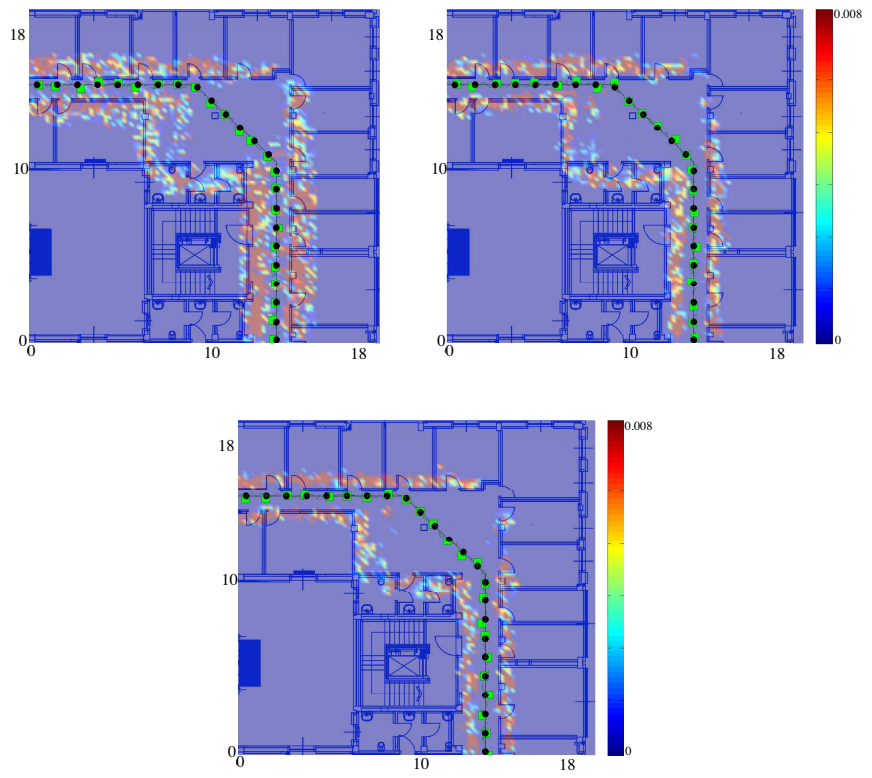

Fig. 11: $2 \mathrm{D}$ mapping using $4 \times 4$ (top-left), $10 \times 10$ (top-right) and $15 \times 15$ (bottom) antenna arrays and with quantization and mobile position $(10 \mathrm{~cm})$ and orientation errors $\left(5^{\circ}\right)$.
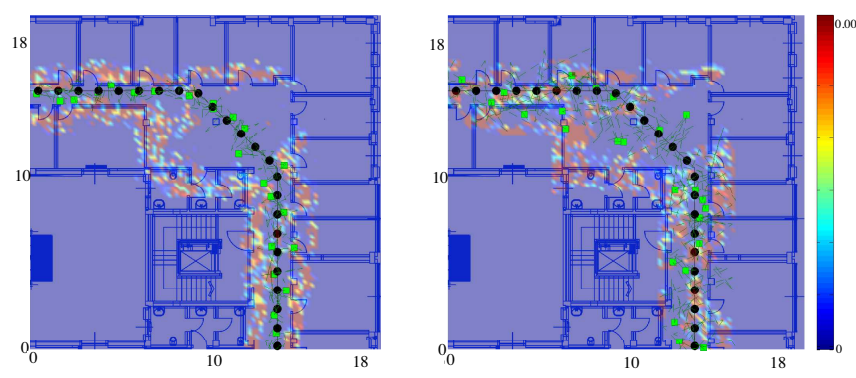

Fig. 12: 2D mapping using $10 \times 10$ antenna arrays with mobile position errors of $0.5 \mathrm{~m}$ (left) and $1 \mathrm{~m}$ (right) and $W=1 \mathrm{GHz}$.

errors might affect the array performance, their negative effect on map reconstruction can be significantly mitigated if their impact on radiation pattern is properly characterized in the measurement model (37) by adopting the analysis of Sec. 3.

In Fig. 11 we include also mobile orientation and position errors caused by an imperfect external positioning system. In particular, we have taken into account orientation estimation errors with standard deviation of $5^{\circ}$, and position estimation errors with $0.1 \mathrm{~m}$ standard deviation [43], both along the $x$ - and the $y$-axis. It is evident from the figure how estimated maps become much more degraded even if the performance of $10 \times 10$ antenna array is still acceptable.

Despite future indoor location systems are expected to guarantee a self-localization with errors below 0.5 $\mathrm{m}$, current technologies provide errors typically greater than $0.5 \mathrm{~m}$. Thus, in Fig. 12 we considered a mobile position error of 0.5 and $1 \mathrm{~m}$, respectively. Performance is evidently degraded, but it could be improved, for
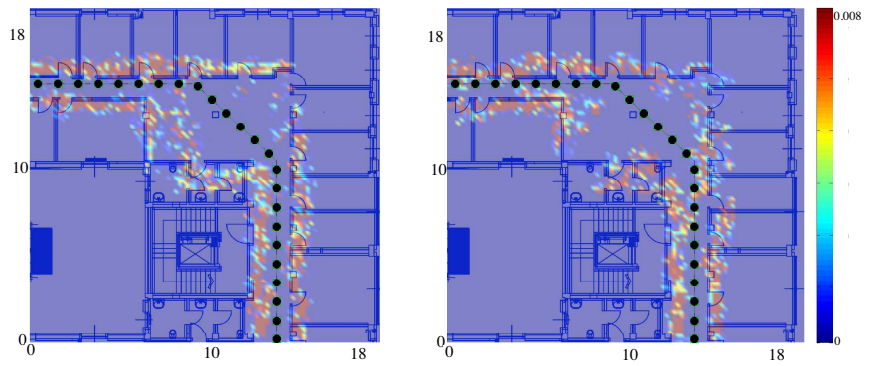

Fig. 13: 2D mapping using $10 \times 10$ antenna arrays with mobile orientation errors of $10^{\circ}$ (left) and $20^{\circ}$ (right) and $W=1 \mathrm{GHz}$.
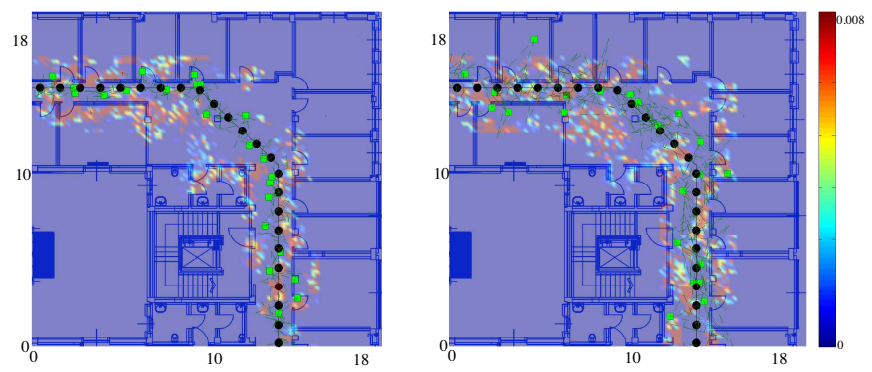

Fig. 14: 2D mapping using $10 \times 10$ antenna arrays with position and mobile orientation errors $0.5 \mathrm{~m}+20^{\circ}$ (left) and $1 \mathrm{~m}+20^{\circ}$ (right) and $W=1 \mathrm{GHz}$.

instance, through crowd sensing mechanism where users share their measurements in a cooperative way.

Fig. 13 reports the mapping results considering an augmented orientation estimation error of $10^{\circ}$ and $20^{\circ}$, respectively. As we can see, the orientation estimation accuracy has a less detrimental effect on the mapping reconstruction with respect to the position.

Finally, in Fig. 14 we reported the results obtained when high position and orientation errors are present. As shown, the mapping quality is highly degraded and a crowd-sensing mechanism becomes essential to refine the map estimation.

\section{Conclusions}

We put forth the idea of adopting massive antenna arrays at $\mathrm{mmW}$ as an effective way to embed personal radar capabilities in future smartphones. The quantization and frequency selectivity effects have been accounted for to derive the pointing error, the beamwidth spread and the ranging accuracy of the personal radar as a function of the number of antenna elements in the array. Our analysis has highlighted how angular resolution, scanning time, signal bandwidth and ranging accuracy are tightly coupled and how a suitable trade-off has to be obtained. Successively, the environment mapping capabilities of the proposed personal radar have been investigated through the introduction of a non-coherent radar architecture and a new state-space model, within a grid-based Bayesian framework, in which each cell of the grid has been characterized by a specific RCS-based 
occupancy value. The peculiarities of the antenna array have been properly exploited by a direct integration of massive array radar measurements with the radar and mapping processes.

To assess the feasibility of the system, simulations have been performed in a realistic indoor scenario. Results have shown that an increased number of array elements guarantees more robustness to non-ideal effects and allows an improved performance in terms of map reconstruction, but it might suffer from a reduced SNR when a constraint on the scanning time is given. Thus, a tradeoff has to be found. In addition, it has been assessed that map reconstruction accuracy is more sensitive to mobile position and orientation perception errors than antenna quantization errors. The latter leads to important implications for the feasibility of indoor mapping with low-cost massive array antennas embedded in nextgeneration smartphones and toward the diffusion of crowd sourcing indoor mapping applications.

\section{APPENDIX A}

To evaluate $\mathbb{E}\left[\delta \Theta_{0}\right]$, consider that in the presence of nonideal effects, the actual pointing direction can be derived by solving

$$
\begin{aligned}
& S\left(\widetilde{\Theta}_{0}, f\right)=\left.\frac{\partial|\widetilde{\mathrm{AF}}(\Theta, f)|^{2}}{\partial \theta}\right|_{\Theta=\widetilde{\Theta}_{0}}=0 \\
& F\left(\widetilde{\Theta}_{0}, f\right)=\left.\frac{\partial|\widetilde{\mathrm{AF}}(\Theta, f)|^{2}}{\partial \phi}\right|_{\Theta=\widetilde{\Theta}_{0}}=0 .
\end{aligned}
$$

Considering the expansion with the first order of the Taylor series evaluated in $\widehat{\Theta}_{0}$, it is

$$
\begin{aligned}
S\left(\widetilde{\Theta}_{0}, f\right) & =S\left(\widehat{\Theta}_{0}+\delta \Theta_{0}, f\right) \\
& =S\left(\widehat{\Theta}_{0}, f\right)+\left.\delta \theta_{0} \frac{\partial S(\Theta, f)}{\partial \theta}\right|_{\Theta=\widehat{\Theta}_{0}}=0 \\
F\left(\widetilde{\Theta}_{0}, f\right) & =F\left(\widehat{\Theta}_{0}+\delta \Theta_{0}, f\right) \\
& =F\left(\widehat{\Theta}_{0}, f\right)+\left.\delta \phi_{0} \frac{\partial F(\Theta, f)}{\partial \phi}\right|_{\Theta=\widehat{\Theta}_{0}}=0 .
\end{aligned}
$$

Thus, considering $\delta \Theta_{0}=\left(\delta \theta_{0}\left(\widehat{\Theta}_{0}, f\right), \delta \phi_{0}\left(\widehat{\Theta}_{0}, f\right)\right)$, and the approach followed in [28], where the derivatives are approximated with their respective mean values, we have

$$
\begin{aligned}
& \delta \theta_{0}\left(\widehat{\Theta}_{0}, f\right)=\left.\left.\frac{-S(\Theta, f)}{\frac{\partial S(\Theta, f)}{\partial \theta}}\right|_{\Theta=\widehat{\Theta}_{0}} \underset{\mathbb{E}\left[\frac{\partial S(\Theta, f)}{\partial \theta}\right]}{\approx}\right|_{\Theta=\widehat{\Theta}_{0}} \\
& \delta \phi_{0}\left(\widehat{\Theta}_{0}, f\right)=\left.\left.\frac{-F(\Theta, f)}{\frac{\partial F(\Theta, f)}{\partial \phi}}\right|_{\Theta=\widehat{\Theta}_{0}} \approx \frac{-F(\Theta, f)}{\mathbb{E}\left[\frac{\partial F(\Theta, f)}{\partial \phi}\right]}\right|_{\Theta=\widehat{\Theta}_{0}}
\end{aligned}
$$

which allow us to write the mean components of $\delta \Theta_{0}$ as

$$
\begin{aligned}
& \mathbb{E}\left[\delta \theta_{0}\left(\widehat{\Theta}_{0}, f\right)\right]=-\left.\frac{\mathbb{E}[S(\Theta, f)]}{\mathbb{E}\left[\frac{\partial S(\Theta, f)}{\partial \theta}\right]}\right|_{\Theta=\widehat{\Theta}_{0}} \\
& \mathbb{E}\left[\delta \phi_{0}\left(\widehat{\Theta}_{0}, f\right)\right]=-\left.\frac{\mathbb{E}[F(\Theta, f)]}{\mathbb{E}\left[\frac{\partial F(\Theta, f)}{\partial \phi}\right]}\right|_{\Theta=\widehat{\Theta}_{0}} .
\end{aligned}
$$

To solve (46), let express $S(\Theta, f)$ as

$$
\begin{aligned}
S(\Theta, f) & =\frac{\partial|\widetilde{\mathrm{AF}}(\Theta, f)|^{2}}{\partial \theta} \\
& =\frac{\partial|\mathrm{AF}(\Theta, f)|^{2}}{\partial \theta}+\frac{\partial \zeta_{\mathrm{err}}(\Theta, f)}{\partial \theta} .
\end{aligned}
$$

By defining

$$
\begin{aligned}
& \frac{\partial \alpha_{m n p q}}{\partial \theta}=k \cos (\theta)\left[(m-p) d_{x} \cos (\phi)+(n-q) d_{y} \sin (\phi)\right] \\
& =\psi_{m n p q}(\Theta, f)
\end{aligned}
$$

it is

$$
\mathbb{E}\left[\frac{\partial \zeta_{\mathrm{err}}(\Theta, f)}{\partial \theta}\right]=j\left[\chi^{2}-1\right] \sum_{m n p q} \psi_{m n p q}(\Theta, f) e^{j \alpha_{m n p q}}
$$

where $\psi_{m n p q}=0$ when $m=p, n=q$. Consequently we have

$$
\mathbb{E}[S(\Theta, f)]=j \chi^{2} \sum_{m n p q} \psi_{m n p q}(\Theta, f) e^{j \alpha_{m n p q}}
$$

from which $\mathbb{E}\left[S\left(\widehat{\Theta}_{0}, f\right)\right]=0$.

Analogously, we can make the same considerations for $\phi$, obtaining $\mathbb{E}\left[\delta \theta_{0}\left(\widehat{\Theta}_{0}, f\right)\right]=\mathbb{E}\left[\delta \phi_{0}\left(\widehat{\Theta}_{0}, f\right)\right]=0$, and consequently $\mathbb{E}\left[\delta \Theta_{0}\right]=0$.

\section{APPENDIX B}

We express $\mathbb{E}\left[\delta \theta_{0}^{2}\left(\widehat{\Theta}_{0}, f\right)\right]$ and $\mathbb{E}\left[\delta \phi_{0}^{2}\left(\widehat{\Theta}_{0}, f\right)\right]$ as

$$
\begin{aligned}
& \mathbb{E}\left[\delta \theta_{0}^{2}\left(\widehat{\Theta}_{0}, f\right)\right]=\left.\frac{\mathbb{E}\left[S^{2}(\Theta, f)\right]}{\left(\mathbb{E}\left[\frac{\partial S(\Theta, f)}{\partial \theta}\right]\right)^{2}}\right|_{\Theta=\widehat{\Theta}_{0}} \\
& \mathbb{E}\left[\delta \phi_{0}^{2}\left(\widehat{\Theta}_{0}, f\right)\right]=\left.\frac{\mathbb{E}\left[F^{2}(\Theta, f)\right]}{\left(\mathbb{E}\left[\frac{\partial F(\Theta, f)}{\partial \phi}\right]\right)^{2}}\right|_{\Theta=\widehat{\Theta}_{0}} .
\end{aligned}
$$

For what the denominator of (51) is regarded, by taking the derivatives of (47), we have:

$$
\begin{aligned}
\frac{\partial S(\Theta, f)}{\partial \theta}= & j \sum_{m n p q} e^{j \alpha_{m n p q}} e^{j\left(\delta_{m n}-\delta_{p q}\right)} \\
& \times\left[\frac{\partial^{2} \alpha_{m n p q}}{\partial \theta^{2}}+j\left(\frac{\partial \alpha_{m n p q}}{\partial \theta}\right)^{2}\right]
\end{aligned}
$$

with

$$
\begin{aligned}
& \frac{\partial^{2} \alpha_{m n p q}}{\partial \theta^{2}}=-k \sin (\theta)\left[(m-p) d_{x} \cos (\phi)+(n-q) d_{y} \sin (\phi)\right] \\
& =\Lambda_{m n p q}(\Theta, f) .
\end{aligned}
$$

Therefore it is

$$
\mathbb{E}\left[\left.\frac{\partial S(\Theta, f)}{\partial \theta}\right|_{\Theta=\widehat{\Theta}_{0}}\right]=j \chi^{2} \sum_{m n p q} \Omega_{m n p q}
$$

where

$$
\Omega_{m n p q}=\Lambda_{m n p q}\left(\widehat{\Theta}_{0}, f\right)+j \psi_{m n p q}^{2}\left(\widehat{\Theta}_{0}, f\right) .
$$


Regarding the numerator of (51) it is

$$
\begin{aligned}
& S^{2}\left(\widehat{\Theta}_{0}, f\right)= \\
& \sum_{m n p q} \sum_{r s t u} \psi_{m n p q}\left(\widehat{\Theta}_{0}, f\right) \psi_{r s t u}\left(\widehat{\Theta}_{0}, f\right) e^{j\left(\delta_{m n}-\delta_{p q}\right)} e^{j\left(\delta_{r s}-\delta_{t u}\right)}
\end{aligned}
$$

whose expectation is given by (57)

$$
\begin{aligned}
& \mathbb{E}\left[S^{2}\left(\widehat{\Theta}_{0}, f\right)\right]=\chi^{4} \sum_{\substack{m n p q \\
m n \neq p q \neq r s \neq t u}} \psi_{m n p q}\left(\widehat{\Theta}_{0}, f\right) \psi_{r s t u}\left(\widehat{\Theta}_{0}, f\right) \\
& +\left(v^{2}-1\right) \sum_{m n p q} \psi_{m n p q}^{2}\left(\widehat{\Theta}_{0}, f\right) \\
& +2 v \chi^{2} \sum_{m n p q} \sum_{\substack{r s \\
m n \neq r s}} \psi_{m n p q}\left(\widehat{\Theta}_{0}, f\right) \psi_{r s p q}\left(\widehat{\Theta}_{0}, f\right) \\
& +2 \chi^{2} \sum_{m n p q} \sum_{\substack{r s \\
p q \neq r s}} \psi_{m n p q}\left(\widehat{\Theta}_{0}, f\right) \psi_{r s m n}\left(\widehat{\Theta}_{0}, f\right)
\end{aligned}
$$

where $v=\mathbb{E}\left[e^{-j 2 \delta_{m n}}\right]=\mathbb{E}\left[e^{j 2 \delta_{m n}}\right]=\frac{1}{\Delta} \sin (\Delta)$. We finally obtain

$$
\begin{aligned}
& \mathbb{E}\left[\delta \theta_{0}^{2}\left(\widehat{\Theta}_{0}, f\right)\right]=\frac{\mathbb{E}\left[S^{2}\left(\widehat{\Theta}_{0}, f\right)\right]}{-\chi^{4}\left[\sum_{m n p q} \Omega_{m n p q}\right]^{2}} \\
& \mathbb{E}\left[\delta \phi_{0}^{2}\left(\widehat{\Theta}_{0}, f\right)\right]=\frac{\mathbb{E}\left[F^{2}\left(\widehat{\Theta}_{0}, f\right)\right]}{-\chi^{4}\left[\sum_{m n p q} \Omega_{m n p q}^{\prime}\right]^{2}}
\end{aligned}
$$

with

$$
\mathbb{E}^{2}\left[\left.\frac{\partial S(\Theta, f)}{\partial \phi}\right|_{\Theta=\widehat{\Theta}_{0}}\right]=-\chi^{4}\left[\sum_{m n p q} \Omega_{m n p q}^{\prime}\right]^{2} .
$$

\section{ApPEndiX C}

Equation (14) can be rearranged by developing the second-order series expansion of the term $e^{j\left[(m-p) \Psi_{\delta_{x}}+(n-q) \Psi_{\delta_{y}}\right]}$ as

$$
\sum_{m n p q} \hat{w}_{m n p q} e^{j \delta_{m n p q}} \Delta_{m n p q}^{2}=\sum_{m n p q} \hat{w}_{m n p q} e^{j \delta_{m n p q}}
$$

where $\Delta_{m n p q}=(m-p) \Psi_{\delta x}+(n-q) \Psi_{\delta y}$, and $\delta_{m n p q}=$ $\delta_{m n}-\delta_{p q}$. To derive the BSA we adopt an approach similar to that proposed in [29] and [44] where the beam is considered alternatively lying on the $X Z$ - and $Y Z$ plane. Specifically, consider the case in which $\tilde{\phi}_{0}=0$ and $\delta \phi=0$ so that the beam lies on the $X Z$-plane, with deviation $\pm \delta \tilde{\theta}_{x 0}$ from the beam pointing direction in that plane. We have that $\Delta_{m n p q}=(m-p) k d_{x} \delta \tilde{\theta}_{x 0} \cos \left(\tilde{\theta}_{0}\right)$ and (60) can be written as

$$
\sum_{m n p q} \hat{w}_{m n p q} e^{j \delta_{m n p q}}(m-p)^{2} k^{2} d_{x}^{2} \delta \tilde{\theta}_{x 0}^{2} \cos ^{2}\left(\tilde{\theta}_{0}\right)=\left|\operatorname{AF}\left(\widetilde{\Theta}_{0}, f\right)\right|^{2} .
$$

It is possible to write

$$
\begin{aligned}
& \mathbb{E}\left[\delta \tilde{\theta}_{x 0}\left(\widetilde{\Theta}_{0}, f\right)\right] \approx \\
& \mathbb{E}\left[ \pm\left[\frac{\left|\operatorname{AF}\left(\widetilde{\Theta}_{0}, f\right)\right|^{2}}{\sum_{m n p q} \hat{w}_{m n p q} e^{j \delta_{m n p q}}(m-p)^{2} k^{2} d_{x}^{2} \cos ^{2}\left(\tilde{\theta}_{0}\right)}\right]^{1 / 2}\right],
\end{aligned}
$$

and analogously

$$
\begin{aligned}
& \mathbb{E}\left[\delta \tilde{\theta}_{y 0}\left(\widetilde{\Theta}_{0}, f\right)\right] \approx \\
& \mathbb{E}\left[ \pm\left[\frac{\left|\operatorname{AF}\left(\widetilde{\Theta}_{0}, f\right)\right|^{2}}{\sum_{m n p q} \hat{w}_{m n p q} e^{j \delta_{m n p q}}(n-q)^{2} k^{2} d_{x}^{2} \cos ^{2}\left(\tilde{\theta}_{0}\right)}\right]^{1 / 2}\right] .
\end{aligned}
$$

Solving (62) and (63) appears very complicated because the expected value regards the ratio of dependent RVs. Nonetheless, they are useful to drastically facilitate numerical calculation of the solution, as it will be done in Sec. 5.2.

\section{RefERENCES}

[1] C. Floerkemeier et al., Eds., The Internet of Things, First Int. Conf. Springer, 2008.

[2] J. Gjengset et al., "Phaser: Enabling phased array signal processing on commodity WiFi access points," in Proc. of the 20th Annual Int.Conf. on Mobile Computing and Networking, ser. MobiCom '14, New York, NY, USA, 2014, pp. 153-164.

[3] F. Adib and D. Katabi, "See through walls with wifi!" in Proc. of the ACM SIGCOMM Conf., ser. SIGCOMM '13, New York, NY, USA, 2013, pp. 75-86.

[4] http://robotics.ccny.cuny.edu/web/.

[5] W. Morris et al., "3D indoor mapping for micro-uavs using hybrid range finders and multi-volume occupancy grids," in RSS 2010 workshop on RGB-D: Advanced Reasoning with Depth Cameras, 2010, Zaragoza, Spain.

[6] H. Durrant-Whyte and T. Bailey, "Simultaneous localisation and mapping (SLAM): Part I the essential algorithms," IEEE Robotics and Automation Mag., vol. 2, pp. 99-110, 2006.

[7] T. Rappaport et al., in Millimeter Wave Wireless Communications. Prentice Hall, 2014.

[8] R. Daniels and R. Heath, "60 GHz wireless communications: emerging requirements and design recommendations," IEEE Mag. Veh. Tech., vol. 2, no. 3, pp. 41-50, 2007.

[9] F. Giannetti, M. Luise, and R. Reggiannini, "Mobile and personal communications in $60 \mathrm{GHz}$ band: A survey," Wirelesss Personal Comunications, vol. 10, pp. 207-243, 1999.

[10] F. Boccardi et al., "Five disruptive technology directions for 5G," IEEE Commun. Mag., vol. 52, no. 2, pp. 74-80, Feb. 2014.

[11] W. Hong et al., "Study and prototyping of practically largescale mmWave antenna systems for 5G cellular devices," IEEE Commun. Mag., vol. 52, no. 9, pp. 63-69, Sep. 2014.

[12] H. Kaouach et al., "Wideband low-loss linear and circular polarization transmit-arrays in V-Band," IEEE Trans. Antennas and Propag., vol. 59, no. 7, pp. 2513-2523, July 2011.

[13] M. W. M. G. Dissanayake et al., "A solution to the simultaneous localization and map building (SLAM) problem," IEEE Trans. Robotics and Automation, vol. 17, pp. 229-241, 2001.

[14] E. Jose and M. Adams, "Relative radar cross section based feature identification with millimeter wave radar for outdoor SLAM," in Proc. IEEE/RSJ Int. Conf. on Intelligent Robots and Systems (IROS), vol. 1, Sept 2004, pp. 425-430 vol.1.

[15] _ - "An augmented state SLAM formulation for multiple lineof-sight features with millimetre wave radar," in Proc. IEEE Int. Conf. on Intelligent Robots and Systems (IROS), Aug 2005, pp. 30873092. 
[16] E. Jose et al., "Predicting Millimeter Wave Radar Spectra for Autonomous Navigation," IEEE Sensors J., vol. 10, no. 5, pp. 960971, May 2010.

[17] F. Guidi, A. Guerra, and D. Dardari, "Millimeter-wave massive arrays for indoor slam," in Proc. IEEE Int. Conf. on Comm. (ICC), June 2014, Sydney, Aus.

[18] A. Guerra, F. Guidi, and D. Dardari, "Millimeter-wave personal radars for 3D environment mapping," in Proc. IEEE Asilomar Conf. on Signals, Systems, and Computers, Pacific Grove, USA, Nov. 2014.

[19] C. Wu, Z. Yang, and Y. Liu, "Smartphones based crowdsourcing for indoor localization," IEEE Trans. Mobile Computing, vol. PP, no. 99, pp. 1-1, 2014.

[20] A. Guerra et al., "Application of transmitarray antennas for indoor mapping at millimeter-waves," in Proc. IEEE European Conf. on Networks and Commun. (EUCNC), 2015.

[21] M. Alzantot and M. Youssef, "Crowdinside: automatic construction of indoor floorplans," in Proc. 20th Int. Conf. on Advances in Geographic Information Systems. ACM, 2012, pp. 99-108.

[22] A. Rai et al., "Zee: zero-effort crowdsourcing for indoor localization," in Proc. 18th annual Int. Conf. on Mobile computing and networking. ACM, 2012, pp. 293-304.

[23] Y. Xuan, R. Sengupta, and Y. Fallah, "Crowd sourcing indoor maps with mobile sensors," in Mobile and Ubiquitous Systems: Computing, Networking, and Services, ser. Lecture Notes of the Institute for Computer Sciences, Social Informatics and Telecommunications Engineering, P. Senac, M. Ott, and A. Seneviratne, Eds. Springer Berlin Heidelberg, 2012, vol. 73, pp. 125-136.

[24] R. Faragher, C. Sarno, and M. Newman, "Opportunistic radio SLAM for indoor navigation using smartphone sensors," in Proc. IEEE Position Location and Navigation Symp. (PLANS), April 2012, pp. $120-128$.

[25] https://www.google.com/ atap/projecttango/.

[26] A. Guerra, F. Guidi, and D. Dardari, "Position and orientation error bound for wideband massive antenna arrays," in Proc. IEEE Int. Conf. on Commun. (ICC), ANLN Workshop, 2015.

[27] J. Ruze, "The effect of aperture errors on the antenna radiation pattern," Il Nuovo Cimento, vol. 9, no. 3, pp. 364-380, 1952. [Online]. Available: http://dx.doi.org/10.1007/BF02903409

[28] L. Rondinelli, "Effects of random errors on the performance of antenna arrays of many elements," in IRE Int. Convention Record, vol. 7, March 1959, pp. 174-189.

[29] C. Balanis, Antenna Theory, 3rd ed. Wiley, 2005.

[30] J. Krieger, C.-P. Yeang, and G. Wornell, “Dense delta-sigma phased arrays," IEEE Trans. Antennas Propag., vol. 61, no. 4, pp. 1825-1837, 2013.

[31] D. Dardari et al., "Ranging with ultrawide bandwidth signals in multipath environments," Proc. of IEEE, Special Issue on UWB Technology E Emerging Applications, vol. 97, no. 2, pp. 404-426, Feb 2009

[32] P. Baltus, P. Smulders, and Y. Yu, Systems and Architectures for Very High Frequency Radio Links. Springer Netherlands, 2008.

[33] W. Wagner, "Radiometric calibration of small-footprint fullwaveform airborne laser scanner measurements: Basic physical concepts," $\{$ ISPRS $\}$ Journal of Photogrammetry and Remote Sensing, vol. 65, no. 6, pp. $505-513,2010$.

[34] K. Sarabandi, E. Li, and A. Nashashibi, "Modeling and measurements of scattering from road surfaces at millimeter-wave frequencies," IEEE Trans. Antennas Propag., vol. 45, no. 11, pp. 1679-1688, Nov. 1997.

[35] D. Dardari, P. Closas, and P. Djuric, "Indoor tracking: Theory, methods, and technologies," IEEE Trans. Veh. Technol., vol. 64, no. 4, pp. 1263-1278, April 2015.

[36] H. Urkowitz, "Energy detection of unknown deterministic signals," Proc. IEEE, vol. 55, no. 4, pp. 523-531, Apr. 1967.

[37] K. M. Wurm, C. Stachniss, and G. Grisetti, "Bridging the gap between feature- and grid-based SLAM," Robotics and Autonomous Systems, vol. 58, no. 2, pp. 140-148, 2010.

[38] O. Ozisik and S. Yavuz, "An occupancy grid based SLAM method," in Proc. IEEE Int. Conf. on Computational Intell. for Measurement Syst. and Applicat. (CIMSA), July 2008, pp. 117-119.

[39] S. Y. Chun and A. Shapiro, "Normal versus noncentral chi-square asymptotics of misspecified models," Multivariate Behavioral Research, vol. 44, no. 6, pp. 803-827, 2009.

[40] V. Degli-Esposti et al., "Measurement and modelling of scattering from buildings," IEEE Trans. Ant. Propag., vol. 55, no. 1, pp. 143153, 2007.
[41] L. Correia and P. Frances, "Estimation of materials characteristics from power measurements at $60 \mathrm{GHz}$," in Proc. IEEE 5th Int. Symposium on Personal, Indoor and Mobile Radio Commun. (PIRMC), 1994, pp. 510-513 vol.2.

[42] FCC, "Revision of Part 15 of the Commissions Rules Regarding Operation in the 57-64 GHz Band. August 2013."

[43] J. Xiong and K. Jamieson, "Arraytrack: A fine-grained indoor location system," in Proc. 10th USENIX Symp. on Networked Syst. Design and Implementation (NSDI). Lombard, IL: USENIX, 2013, pp. 71-84.

[44] R. S. Elliot, Antenna Theory and Design. New York: Prentice-Hall, 1981.

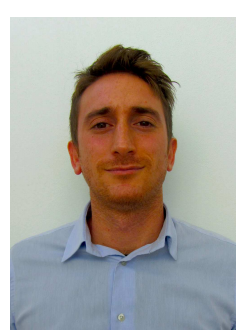

Francesco Guidi (S'09 - M'14) received the B.S. degree and the M.S. degree, with honors, from the University of Bologna in biomedical (2006) and in electronics and telecommunications (2009) engineering, respectively. In 2013 he received the PhD degree both from Ecole Polytechnique ParisTech, France (Computer Science Specialty) and from University of Bologna, Italy, in electronics, telecommunications and information technologies.

$\mathrm{He}$ is currently a postdoctoral researcher at University of Bologna. He received the best student paper award at the 2014 IEEE International Conference on Ultra-Wideband. His research interests include RFID technology, joint antenna and channel characterization, signal processing.

Dr. Guidi serves as reviewer for numerous IEEE Journals and Conferences and was a member of the local organization committee for the 2011 IEEE International Conference on Ultra-Wideband.

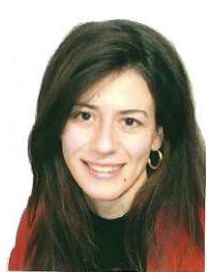

Anna Guerra $\left(S^{\prime} 13\right)$ received the Laurea degree in electronics and telecommunications engineering from the University of Bologna in 2011. She is currently a Ph.D. candidate in electronics, telecommunications and information technologies at University of Bologna. In 2012, she was a Research Assistant in a collaboration project between the Consorzio Nazionale Interuniversitario per le Telecomunicazioni (CNIT) and French Atomic Energy Commission (CEALETI), Grenoble, France. In 2014, she was visiting student at CEA-LETI. She received the best student paper award at the 2014 IEEE International Conference on Ultra-Wideband in Paris (France). Her research interest includes wireless sensor networks, radio localization, array signal processing, UWB and millimeter-wave technology. 


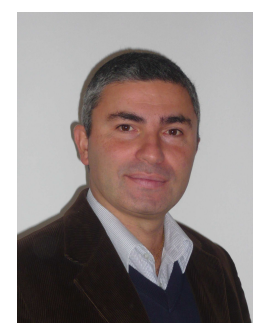

Davide Dardari (M'95-SM'07) received the Laurea degree in electronic engineering (summa cum laude) and the $\mathrm{PhD}$ degree in electronic engineering and computer science from the University of Bologna, Italy, in 1993 and 1998, respectively.

$\mathrm{He}$ is an Associate Professor at the University of Bologna at Cesena, Italy, where he participates with WiLAB (Wireless Communications Laboratory). Since 2005, he has been a Research Affiliate at Massachusetts Institute of Technology (MIT), Cambridge, USA. He is also Research Affiliate at IEIIT/CNR (National Research Council) and CNIT (Consorzio Nazionale Interuniversitario per le Telecomunicazioni). He published more than 150 technical papers and played several important roles in various $\mathrm{Na}$ tional and European Projects. His interests are on ultra-wide bandwidth (UWB) systems, ranging and localization techniques, distributed signal processing, as well as wireless sensor networks. He received the IEEE Aerospace and Electronic Systems Society's M. Barry Carlton Award (2011) and the IEEE Communications Society Fred W. Ellersick Prize (2012).

Prof. Dardari is Senior Member of the IEEE where he was the Chair for the Radio Communications Committee of the IEEE Communication Society. He was co-General Chair of the 2011 IEEE International Conference on Ultra-Wideband and co-organizer of the IEEE International Workshop on Advances in Network Localization and Navigation (ANLN) - ICC 2013/2014/2015. He was also co-Chair of the Wireless Communications Symposium of the 2007 IEEE International Conference on Communications, and co-Chair of the 2006 IEEE International Conference on Ultra-Wideband. He served as Lead Editor for the EURASIP Journal on Advances in Signal Processing (Special Issue on Cooperative Localization in Wireless Ad Hoc and Sensor Networks), Guest Editor for Proceedings of IEEE (Special Issue on UWB Technology \& Emerging Applications), for the Physical Communication Journal (ELSEVIER) (Special Issue on Advances in UWB Wireless Communications) and for IEEE TRANSACTIONS ON VEHICULAR TECHNOLOGY (Special Session on indoor localization, tracking, and mapping with heterogeneous technologies). He served as an Editor for IEEE TRANSACTIONS ON WIRELESS COMMUNICATIONS from 2006 to 2012. 